\title{
Modeling a photoinduced planar-to-homeotropic anchoring transition triggered by surface azobenzene units in a nematic liquid crystal
}

\author{
Patrick Oswald ${ }^{1, *}$ and Jordi Ignés-Mullol ${ }^{1,2,3}$ \\ ${ }^{1}$ Univ Lyon, ENS de Lyon, Univ Claude Bernard, CNRS, Laboratoire de Physique, F-69342 Lyon, France \\ ${ }^{2}$ Institute of Nanoscience and Nanotechnology (IN2UB), Universitat de Barcelona, Barcelona, Spain \\ ${ }^{3}$ Departament de Ciència de Materials i Química Física, Universitat de Barcelona, Martí i Franquès 1, 08028, Barcelona, Spain
}

(Received 29 June 2017; published 25 September 2017)

\begin{abstract}
The performance of light-controlled liquid crystal anchoring surfaces depends on the nature of the photosensitive moieties and on the concentration of spacer units. Here, we study the kinetics of photosensitive liquid crystal cells that incorporate an azobenzene-based self-assembled monolayer. We characterize the photoinduced homeotropic-to-planar transition and the subsequent reverse relaxation in terms of the underlying isomerization of the photosensitive layer. We show that the response time can be precisely adjusted by tuning the lateral packing of azobenzene units by means of inert spacer molecules. Using simple kinetic assumptions and a well-known model for the energetics of liquid crystal anchoring we are able to capture the details of the optical microscopy experimental observations. Our analysis provides fitted values for all the relevant material parameters, including the zenithal and the azimuthal anchoring strength.
\end{abstract}

DOI: 10.1103/PhysRevE.96.032704

\section{INTRODUCTION}

It is well known that the contact of a nematic liquid crystal (LC) with any surface influences the orientation of its director field. This phenomenon is known as the anchoring of the LC on the surface, and its study is relevant both for fundamental purposes and for the development of LC-based devices. The chemical composition and the symmetries of the surface determine preferred anchoring directions for the LC molecules, both with respect to the surface normal (zenithal anchoring) and to an in-plane projection (azimuthal anchoring) [1]. The strength of this anchoring, expressed in terms of the energy cost per unit area for the LC molecules to deviate from the preferred direction, determines whether the molecular orientation is permanently fixed (strong anchoring) or whether it can be easily overcome with external influences (weak anchoring). The anchoring can be monostable or multistable, depending on whether there is only one or several optimal orientations for the director, and its character can change as a function of experimental parameters such as temperature, material flow, electric field, humidity, etc., sometimes leading to anchoring transitions [2].

The possibility to exert a reversible control on the anchoring conditions allows to develop responsive devices such as optical switches or microactuators [3-6]. This is a different strategy than applying the external influence directly on the LC, offering an enhanced versatility in the choice of mesogens. Among the available control methods, the use of light and photosensitive anchoring layers offers a fast and robust approach [7]. For instance, polyimide compounds that incorporate azobenzene moieties can be used to prepare thin films that align with polarized light (often in the UV band) and can be subsequently fixed by thermal annealing [8]. A different strategy is to adsorb small photosensitive molecules on the control surface. Ichimura et al. pioneered the use of LC

\footnotetext{
*patrick.oswald@ens-lyon.fr
}

anchoring surfaces based on monomolecular layers of azobenzene derivatives [9]. A well-tested practical realization consists on using elongated molecules that feature a triethoxysilane head group and an azobenzene moiety within an alkane chain. Such compounds bind covalently to glass or oxide surfaces, and allow to take advantage of the light-induced cis-trans isomerization of the azobenzene group. Planar anchoring of the LC is induced for the cis-rich surface, achievable under UV irradiation, while homeotropic anchoring is promoted by the trans-rich surface, which is obtained under thermal relaxation in the dark (typically slow) or forced by irradiation with blue or green light. Similar results have been demonstrated with different photosensitive molecules, sometimes with the photosensitive moieties adsorbing from the bulk [10-12] rather than being grafted as a stable film. All approaches lead to a rather general effect, although the obtained anchoring may be tilted rather than planar, with surfaces featuring varying degrees of stability. Light-induced changes depend on the type of LC used but also on the nature and the density of the azobenzene units attached to the surface [13-15].

Light control of the LC anchoring conditions has the significant advantage of allowing local addressability. This way, selected areas in a sample can be patterned with different orientations of a planar director field [5,6], or regions with homeotropic and planar anchoring can be made to coexist $[6,12]$. This characteristic was recently used to study the assembly of micrometer-sized particles and drops dispersed in a nematic LC and their transport along arbitrary paths that were imprinted by irradiation with either UV or blue light [6]. An important parameter in such experiments is the time a planar path survives once it has been created. Indeed, the photoinduced planar alignment within the path becomes unstable after some time because of the progressive and spontaneous relaxation of the azobenzene anchoring monolayer from its cis into its more stable trans isomeric form.

In this paper, we quantitatively study the kinetics of lightinduced anchoring transitions and the anchoring energetics of 
a LC aligning layer prepared with self-assembled monolayers of a mixture of photosensitive and nonphotosensitive silanes. After this introduction, we begin with a detailed account of the experimental procedures. The article follows with a experimental study of the spontaneous planar to homeotropic relaxation after UV irradiation of the LC cells. Based on these data, we propose a model for the observed behavior based on a combination of anchoring energy functionals and the kinetics of the cis-trans isomerization. This allows to relate the intrinsic temperature-dependent relaxation time to surface energetics parameters. We complete the analysis with a measurement of the zenithal and azimuthal anchoring energy, and describe a protocol that allows to overcome the memorization of an easy axis in the planar orientation.

\section{SAMPLE PREPARATION AND EXPERIMENTAL SETUP}

We prepared self-assembled monolayers with a mixture of a photosensive and a nonphotosensitive silane. The photosensitive compound was (E)-4-(4-((4-octylphenyl) diazenyl)phenoxy)- $N$-(3-(triethoxysilyl)propyl)butanamidehenceforth called AS-which was custom-synthesized by GalChimia, Spain. The compound was grafted on glass surfaces following well-known procedures [16]. In short, the deposition solution was prepared by dissolving AS and $n$-butylamine (Sigma-Aldrich; used as a catalyzer) in toluene (99\%, Sigma-Aldrich) at a mass ratio 1:7:173. To this solution, an amount of ehtyltriethoxysilane (from Sigma-Aldrich, Germany)—henceforth called ES-was added. The molar ratio of ES with respect to the total moles of silane,

$$
C_{\mathrm{ES}}=\frac{n_{\mathrm{ES}}}{n_{\mathrm{ES}}+n_{\mathrm{AS}}},
$$

was varied between 0 and 0.9 .

The deposition was made on soda lime glass slides (Duran Group, Germany), cleaned with soap, chromosulfuric acid, rinsed with distilled water, and dried under a stream of nitrogen. The glass plates and the deposition solution were placed inside a hermetic glass tube and warmed to $80^{\circ} \mathrm{C}$ for $2 \mathrm{~h}$. The plates where subsequently sonicated for $2 \mathrm{~min}$ at room temperature. Upon removal, the slides were quickly rinsed with toluene to avoid precipitation of the solute, followed by a 10-min wash with toluene under ultrasounds. Finally, the plates were dried under a stream of nitrogen and immediately glued together along two nickel wires of diameter $21 \mu \mathrm{m}$ used as a spacer. For hybrid samples, one plate was treated for strong homeotropic anchoring with the polyimide Nissan 0626. In this case, the polyimide was deposited by spin-coating, prebaked at $80^{\circ} \mathrm{C}$ for $1 \mathrm{~min}$, and baked at $180^{\circ} \mathrm{C}$ for $1 \mathrm{~h}$. For strong planar anchoring, the polyimide compound Nissan 0825 was used. In this case, the polyimide was deposited by spin-coating, prebaked at $80^{\circ} \mathrm{C}$ for $1 \mathrm{~min}$, and baked at $280^{\circ} \mathrm{C}$ for $1 \mathrm{~h}$, followed by gentle unidirectional rubbing with a velvet cloth. Once the glue was set, the exact gap thickness was measured with a spectrometer and the sample was filled with the nematic phase.

The chosen LC was CCN-37 ( $4 \alpha, 4^{\prime} \alpha$-propylheptyl- $1 \alpha, 1^{\prime} \alpha$ bicyclohexyl- $4 \beta$-carbonitrile from Nematel, Germany). This LC has a nematic phase between $22.5^{\circ} \mathrm{C}$ and $54.1^{\circ} \mathrm{C}$ [17].
It has a very small birefringence $[17,18]$, which made the tilt angle measurements easier. More important, it has a negative magnetic anisotropy $[17,19]$, a rare property for a calamitic molecule. As a consequence, the CCN-37 molecules align in a plane perpendicular to the magnetic field. We took advantage of this property while studying the photoinduced homeotropic-to-planar anchoring transition by systematically placing our sample in a strong magnetic field $(B=1 \mathrm{~T})$ parallel to the glass plates. In this way, the director field has a well-known homogeneous azimuth angle while the molecules tilt freely everywhere in the same plane perpendicular to $\vec{B}$ during the transition. All our measurements were performed using the experimental setup described in Ref. [19], to which we refer for a complete description. In short, the sample is placed in homemade thermostated cell, regulated to within $\pm 0.02{ }^{\circ} \mathrm{C}$. The magnetic field is imposed by a Halbach ring, which can rotate around its revolution axis. This allowed us to impose either a fixed magnetic field to the sample or a rotating magnetic field. Finally, the UV irradiation of the sample at a wavelength of $365 \mathrm{~nm}$ was performed with an unpolarized led lamp (Thorlabs M365L2) placed on top of the Halbach ring. In this position, we measured that the UV light power density on the sample surface was about $2 \mathrm{~mW} \mathrm{~cm}^{-2}$. After irradiation, the UV lamp was removed and the sample was observed under the microscope between crossed polarizers. All observations were performed under red monochromatic light $(\lambda=633 \mathrm{~nm})$ to ensure that the photosensitive layer was not perturbed.

\section{PHOTO-INDUCED ANCHORING TRANSITION}

\section{A. Transmittance between crossed polarizers}

We first studied samples prepared between two glass plates treated with the same photosensitive layer. Each sample was characterized by a different concentration of ES, which plays the role of the control parameter. All samples were initially in their stable homeotropic state. After a 2- to 5-min UV irradiation, the director systematically tilted inside the samples, revealing an anchoring transition at the surfaces. To study the tilted state and its stability, we recorded as a function of time-from time $t=0$ at which the UV lamp was switched off-the sample transmittance between crossed polarizers. To observe a signal with a maximum amplitude, the magnetic field was oriented at an angle of $45^{\circ}$ with the polarizers. For this orientation, the transmittance reads

$$
\operatorname{Tr}=\frac{1}{2}(1-\cos \Phi)
$$

where $\Phi$ is the phase shift between the ordinary and extraordinary rays:

$$
\Phi=\frac{2 \pi d \Delta n}{\lambda} \sin ^{2} \theta .
$$

In this formula, which is valid for a low birefringence material such as $\mathrm{CCN}-37, \theta$ is the uniform tilt angle of the director with respect to the normal to the surfaces inside the sample, $d$ is the thickness, $\Delta n$ is the birefringence, and $\lambda$ is the light wavelength (here $633 \mathrm{~nm}$ ).

Figure 1 shows examples of typical transmittance curves measured with a video camera inside a square of side length $100 \mu \mathrm{m}$ at different concentrations and temperatures. All the curves share similar qualitative features: a long plateau 

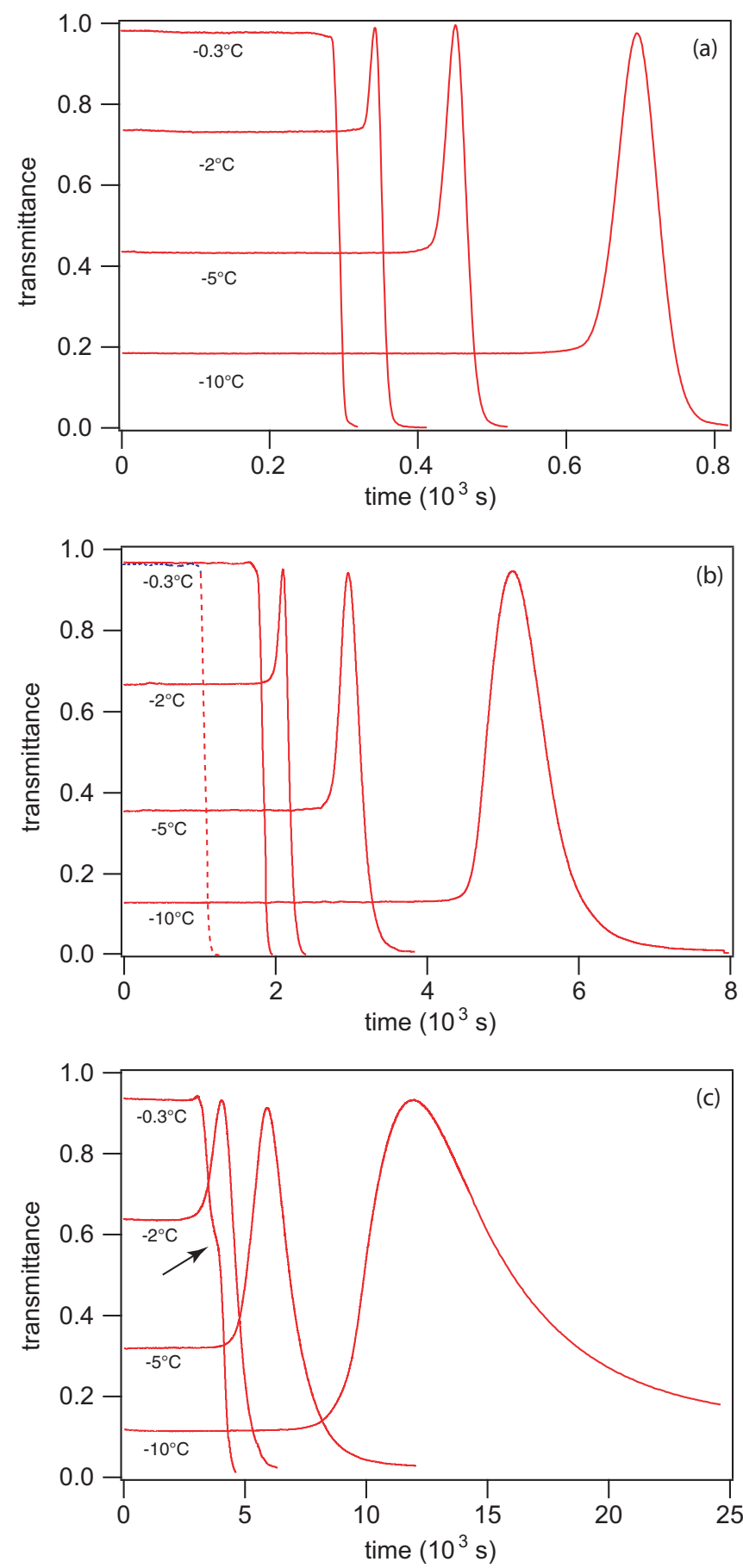

FIG. 1. Examples of transmittance curves as a function of time, with crossed polarizers oriented at $45^{\circ}$ of the magnetic field, for three different concentrations of ES $\left[C_{\mathrm{ES}}=0.218\right.$ (a), 0.817 (b), and 0.847 (c)], and four temperatures $\left(\delta T=T-T_{\mathrm{NI}}=-0.3,-2,-5\right.$, and $-10^{\circ} \mathrm{C}$ ). The sample thickness is $d=20.68 \mu \mathrm{m}$ (a), $21.63 \mu \mathrm{m}$ (b), and $21.87 \mu \mathrm{m}$ (c). All the curves were measured in the saturation regime after a long UV exposure time $t_{\mathrm{UV}}$ ranging between 120 and $300 \mathrm{~s}$, except the dashed line in (b) for which $t_{\mathrm{UV}}=22 \mathrm{~s}$.

on which the transmittance is constant followed by a rapid variation until the complete extinction when the sample is again homeotropic. Each curve is characterized by three quantities: the height of the plateau, which is related to the tilt

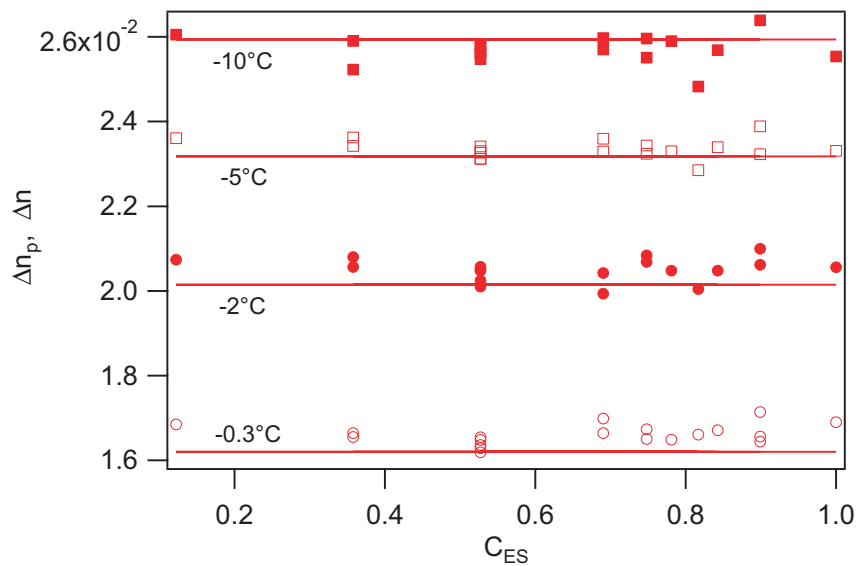

FIG. 2. Effective birefringence (symbols) measured at four different temperatures on the plateau of the transmittance curves as a function of the ES concentration. The horizontal solid lines give the values of the birefringence measured with a tilting compensator in a conventional planar sample (rubbed polyimide).

angle $\theta_{p}$ at the plateau, time $t p_{1}$, the duration of the plateau, and time $t p_{2}$ at which the homeotropic state is recovered. We measured these parameters for each curve.

First, we measured the tilt angle at the $c i s$-rich plateau. From the measurement of the plateau transmittance, we deduced the effective birefringence: $\Delta n_{p}=\Delta n \sin ^{2} \theta_{p}=(\lambda / d)[1-$ $\left.(1 / 2 \pi) \arccos \left(1-2 T_{r}\right)\right]$ (Fig. 2). We found that, within our experimental errors, $\Delta n_{p}$ does not depend on $C_{\mathrm{ES}}$. We also measured independently the birefringence $\Delta n$ of CCN-37 at $\lambda=633 \mathrm{~nm}$ by employing the same methods as in Ref. [18]. The corresponding values of $\Delta n$ are reported in Fig. 2 (horizontal solid lines). As we can see, $\Delta n_{p}=\Delta n$ within the experimental errors, showing that the anchoring is planar on the plateau $\left(\theta_{p}=90^{\circ}\right.$ to within a few degrees $)$. Note that the anchoring remains planar at $C_{\mathrm{ES}}=1$ when the sample is only treated with ES.

Second, we measured the plateau duration. It is important to note here that this quantity is independent of the UV exposure time $t_{\mathrm{UV}}$ provided the latter is large enough. This is visible in Fig. 3 measured with the same sample as in Fig. 1(b) at $\delta T=-0.3^{\circ} \mathrm{C}$. This curve shows that, for $t_{\mathrm{UV}}<7 \mathrm{~s}$, there is no plateau. On the other hand, a plateau develops when $t_{\mathrm{UV}}>7 \mathrm{~s}$ as we can see in Fig. 1(b) where the dashed line curve corresponds to $t_{\mathrm{UV}}=22 \mathrm{~s}$. In this regime, the plateau duration (denoted by $t p_{1}$ in the following) increases monotonically from 0 to a constant value as a function of $t_{\mathrm{UV}}$. The fact that the plateau duration tends to a constant at large $t_{\mathrm{UV}}$ means that the concentration of $c i s$-isomer saturates on the surfaces when $t_{\mathrm{UV}}$ is long enough. This is the saturation regime in which our subsequent experiments were performed. We found that $t p_{1}$ strongly depends on $C_{\mathrm{ES}}$ as shown in Fig. 4. In this figure, each point represents an average over two to five measurements realized with different samples (about 40 in total). The typical error for each point is about $\pm 20 \%$. This graph shows that $t p_{1}$ is an increasing function of the $C_{\mathrm{ES}}$ that diverges for a critical concentration close to 0.84 . Above this concentration, the anchoring is no longer homeotropic at equilibrium but conical, until it becomes planar for $C_{\mathrm{ES}}=1$. Note that the 


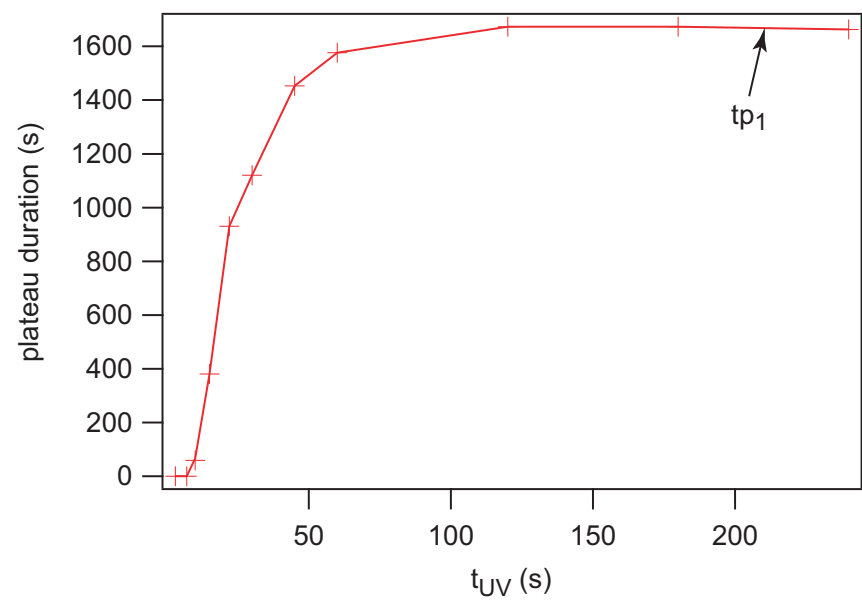

FIG. 3. Plateau duration $\left(t p_{1}\right)$ as a function of the UV exposure time. $d=21.63 \mu \mathrm{m}, C_{\mathrm{ES}}=0.817$, and $\delta T=-0.3{ }^{\circ} \mathrm{C}$.

critical concentration slightly depends on temperature. This is visible in Fig. 1(c) corresponding to $C_{\mathrm{ES}}=0.847$. For this concentration, the transmittance curve tends to 0 at long time at $\delta T=-0.3,-2$, and $-5^{\circ} \mathrm{C}$, but to a finite value close to 0.15 at $\delta T=-10^{\circ} \mathrm{C}$, which corresponds to a director tilt angle $\theta \approx 22^{\circ}$. This means that for this sample, the equilibrium anchoring is no longer homeotropic at low temperature but conical.

Third, we measured at which time $t p_{2}$ the homeotropic state is recovered. This time is more difficult to measure than $t p_{1}$ because of a frequent artifact which is visible in Fig. 1(c). On this example, a kind of shoulder (indicated by an arrow) is
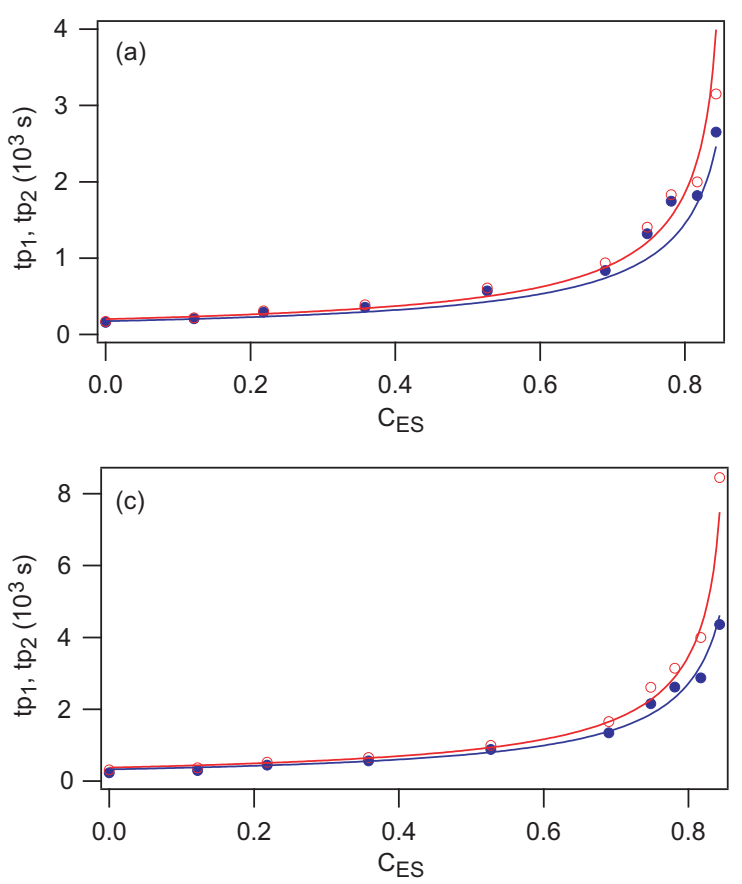

clearly visible on the curve measured at $\delta T=-0.3^{\circ} \mathrm{C}$, but it is not observed on the analogous curves shown in Figs. 1(a) and 1(b). This shoulder is due to the fact that, in this sample, the time at which the anchoring ceases to be planar is not exactly the same on the two plates. This was difficult to avoid in spite of the two plates being always prepared simultaneously in the same silane solution. The presence of this shoulder leads to an overestimation of $t p_{2}$. This is the reason why we did not measure $t p_{2}$ in the samples in which such artifact was clearly visible. Another difficulty was that the transmittance curve was usually rounded at the end. For this reason, we measured $t p_{2}$ as the time at which the transmittance decreases below 0.05 . Measurements of $t p_{2}$ are shown in Fig. 4.

To complete these measurements, we studied the aging of the photosensitive surface treatment. We found that, in general, the length of the plateau regularly decreases during the weeks that follow the sample preparation. On the other hand, the shape of the transmittance curves remained unchanged as can be seen in Fig. 5. In this figure, two sets of curves are shown: the solid curves were measured in a fresh sample and the dashed curves were measured in the same sample two months later. An important point to emphasize is that the decrease of the plateau duration $t p_{1}$ depends on temperature. For instance, in Fig. $5, t p_{1}$ decreases by $\sim 150 \mathrm{~s}$ at $\delta T=-0.3{ }^{\circ} \mathrm{C}, \sim 190$ $\mathrm{s}$ at $\delta T=-2{ }^{\circ} \mathrm{C}, \sim 290 \mathrm{~s}$ at $\delta T=-5^{\circ} \mathrm{C}$, and $\sim 460 \mathrm{~s}$ at $\delta T=-10^{\circ} \mathrm{C}$. This observation will be discussed in the next section.

\section{B. Model}

To model the reported behavior, we propose that the anchoring potential on the surfaces results from a competition
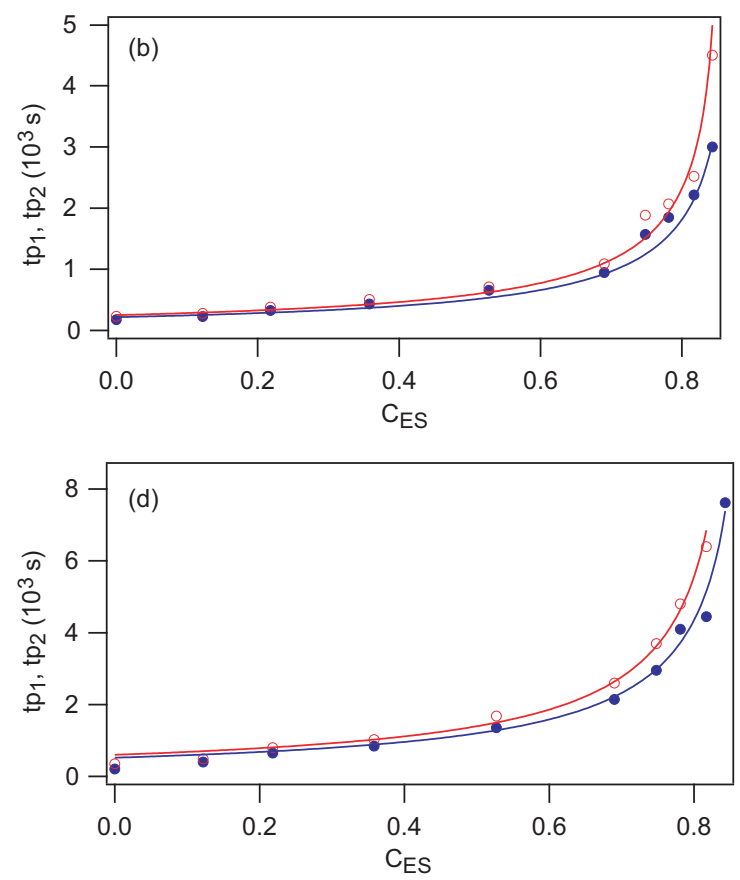

FIG. 4. Plateau duration $t p_{1}$ (blue solid circles) and time $t p_{2}$ (red empty circles) at which the anchoring is again homeotropic as a function of the ES concentration at different temperatures: (a) $\delta T=-0.3{ }^{\circ} \mathrm{C}$; (b) $\delta T=-2{ }^{\circ} \mathrm{C}$; (c) $\delta T=-5^{\circ} \mathrm{C}$; (d) $\delta T=-10^{\circ} \mathrm{C}$. These two times diverge for a concentration close to 0.84 . In these graphs each point represents an average over two to five measurements realized with different samples. The solid lines are the theoretical curves calculated by taking the values of the model parameters given in the text. 


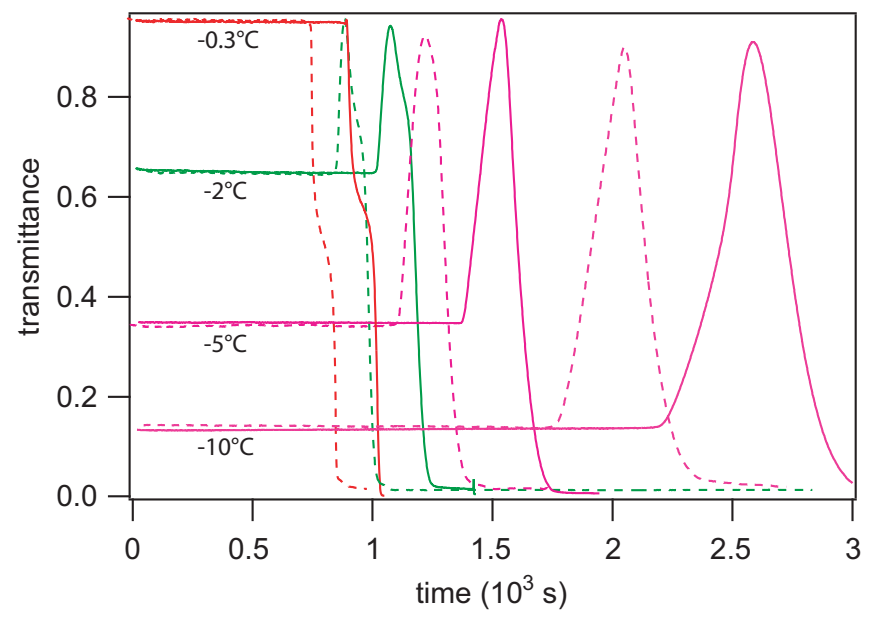

FIG. 5. Transmittance curves measured with a fresh sample (solid lines) and with the same sample stored two months at room temperature (dashed lines). Except for the plateau duration, the curves are essentially the same. $d=21.69 \mu \mathrm{m}$ and $C_{\mathrm{ES}}=0.69$.

between the three kinds of molecules that occupy the surface: the ES molecules and the AS molecules in their cis and trans configurations, respectively. Let $x$ (respectively, $1-x$ ) be the concentration of ES (respectively, AS) molecules attached to the surface. If the surface attachment probability is the same for both types of silane molecules, the bulk and surface compositions must be the same: $x=C_{\mathrm{ES}}$. We make this assumption in the following, knowing that this result was shown by Noonan et al. [16] for self-assembled monolayers pepared with a mixture of alkyl-silanes of different chain lengths.

Each type of molecule is characterized by its own anchoring potential $W$. As recalled in Ref. [20] the symmetry of the surface (revolution) and of the nematic (inversion) imposes that $W(\theta)=W(-\theta)=W(\pi \pm \theta)$ provided that there is no surface polarization, which we assume. In this case, the most general form of $W(\theta)$ is a Fourier expansion in $\cos (2 n \theta)$ with $n=$ $1,2, \ldots$ In the following, we will only keep the first two terms in $\cos (2 \theta)$ and $\cos (4 \theta)$. We have seen that the ES molecules give a planar anchoring. For this reason, we write

$$
W_{\mathrm{ES}}=W_{a}\left[\frac{1}{2} \cos (2 \theta)+\frac{\beta_{\mathrm{ES}}}{4} \cos (4 \theta)\right]
$$

with the anchoring energy $W_{a}>0$ and $\left|\beta_{\mathrm{ES}}\right|<1 / 2$.

For the the AS molecules, we must distinguish the two isomers. For the trans-isomer which gives a homeotropic anchoring, we take

$$
W_{\text {trans }}=W_{a}\left[-\frac{\alpha_{\text {trans }}}{2} \cos (2 \theta)+\frac{\beta_{\text {trans }}}{4} \cos (4 \theta)\right],
$$

with $\alpha_{\text {trans }}>0$ and $\left|\beta_{\text {trans }}\right|<\alpha_{\text {trans }} / 2$ and for the cis-isomer, we take

$$
W_{\text {cis }}=W_{a}\left[\frac{\alpha_{\text {cis }}}{2} \cos (2 \theta)+\frac{\beta_{\text {cis }}}{4} \cos (4 \theta)\right],
$$

with $\alpha_{\text {cis }}>0$ and $\left|\beta_{\text {cis }}\right|<\alpha_{\text {cis }} / 2$, knowing that this isomer favors a planar anchoring.
The next step is to calculate the resulting surface potential. Because of the nematic elasticity, the director field cannot distort at the scale of a few molecular lengths. For this reason $\theta$ is homogeneous on the surface and we assume that the global surface potential is simply a weighted average of the individual potentials:

$W(\theta, t)=(1-x)\left\{y(t) W_{\mathrm{cis}}+[1-y(t)] W_{\text {trans }}\right\}+x W_{\mathrm{ES}}$,

where $y(t)$ is the fraction of $c i s$-isomer in the layer at time $t$ by choosing $t=0$ at the end of the UV exposure.

Let us now calculate $y(t)$. The most simple assumption is to assume that the isomerization process follows a first-order kinetics:

$$
\frac{d y}{d t}=k I(1-y)-\gamma y,
$$

where $I$ is the intensity of the UV light, which is kept constant in our experiments, $k$ is the trans-to-cis constant of isomerization, and $\gamma$ is the rate of spontaneous transformation of the cis-isomer into the more stable trans-isomer. In this equation, the first term in the right-hand side describes the creation of the cis isomer under UV and the second term describes its spontaneous destruction into the more stable trans configuration. This equation shows that under constant UV illumination, the system reaches a stationary state with a concentration of cis isomer given by

$$
y_{\mathrm{sat}}=\frac{k I}{k I+\gamma} \text {. }
$$

In practice, we are in the saturation regime after the UV illumination. This means that at time $t=0, y=y_{\text {sat. }}$. Solving Eq. 8 with this initial condition and $I=0$ gives

$$
y(t)=y_{\text {sat }} \exp \left(-\frac{t}{\tau}\right),
$$

where $\tau=1 / \gamma$ is the cis-to-trans spontaneous relaxation time. As we will see later, the typical creation time under UV of the cis-isomer $\tau_{\mathrm{UV}}=1 /(k I)$ is much smaller than $\tau$, for the used UV light intensity. For this reason, we will take $y_{\text {sat }}=1$ in the following.

We can now calculate the global surface potential. Substitution of $W_{\mathrm{ES}}, W_{\text {trans }}$, and $W_{\text {cis }}$ by their expressions in Eq. (6) gives

$$
W(\theta, t)=W_{a}\left[\frac{\alpha(t)}{2} \cos (2 \theta)+\frac{\beta(t)}{4} \cos (4 \theta)\right],
$$

with

$$
\alpha(t)=x-\alpha_{\text {trans }}(1-x)+y(t)\left(\alpha_{\text {trans }}+\alpha_{\text {cis }}\right)(1-x)
$$

and

$\beta(t)=\beta_{\text {trans }}(1-x)+\beta_{\mathrm{ES}} x+\left(\beta_{\text {cis }}-\beta_{\text {trans }}\right)(1-x) y(t)$.

The azimuthal angle $\theta(t)$ is finally given by minimizing $W(\theta, t)$ at each time.

Next, we show that this model predicts a continuous planarto-homeotropic transition. The anchoring ceases to be planar when the concentration of $\mathrm{cis}$ isomer has decreased down to $y_{1}$ given by $\alpha\left(y_{1}\right)=2 \beta\left(y_{1}\right)$ [20], which yields

$$
y_{1}=\frac{\left(\alpha_{\text {trans }}+2 \beta_{\text {trans }}\right)(1-x)-\left(1-2 \beta_{\mathrm{ES}}\right) x}{(1-x)\left(\alpha_{\text {trans }}+2 \beta_{\text {trans }}+\alpha_{\text {cis }}-2 \beta_{\text {cis }}\right)} .
$$


This happens at a time $t p_{1}$ that satisfies

$$
t p_{1}=-\tau \ln \left(y_{1}\right) .
$$

Similarly, the anchoring is again homeotropic when the concentration of cis isomer satisfies $\alpha\left(y_{2}\right)=-2 \beta\left(y_{2}\right)$ [20], which gives

$$
y_{2}=\frac{\left(\alpha_{\text {trans }}-2 \beta_{\text {trans }}\right)(1-x)-\left(1+2 \beta_{\mathrm{ES}}\right) x}{(1-x)\left(\alpha_{\text {trans }}-2 \beta_{\text {trans }}+\alpha_{\text {cis }}+2 \beta_{\text {cis }}\right)} .
$$

This occurs at a time $t p_{2}$ given by

$$
t p_{2}=-\tau \ln \left(y_{2}\right) \text {. }
$$

Note that, in agreement with experiments, $t p_{1}$ diverges when $y_{1}=0$ at the concentration

$$
x_{1}=\frac{\alpha_{\text {trans }}+2 \beta_{\text {trans }}}{1-2 \beta_{\mathrm{ES}}+\alpha_{\text {trans }}+2 \beta_{\text {trans }}},
$$

while $t p_{2}$ diverges when $y_{2}=0$ at the concentration

$$
x_{2}=\frac{\alpha_{\text {trans }}-2 \beta_{\text {trans }}}{1+2 \beta_{\mathrm{ES}}+\alpha_{\text {trans }}-2 \beta_{\text {trans }}} .
$$

We observe $x_{2}<x_{1}$ (Fig. 4), which imposes $\beta_{\text {trans }}+$ $\alpha_{\text {trans }} \beta_{\mathrm{ES}}>0$. We emphasize that the anchoring at equilibrium (when $I=0$ ) is homeotropic when $x<x_{2}$, tilted when $x_{2}<$ $x<x_{1}$ and planar when $x>x_{1}$.

This model can also be used to relate the plateau duration $t_{\text {plateau }}$ as a function of the UV exposure time $t_{\mathrm{UV}}$ in the unsaturated regime (Fig. 3). It is important to stress that, after relaxation following UV irradiation, the sample goes back to being homeotropic when $y<y_{2}$. Although $y_{2}>0$, no changes are detectable during the subsequent relaxation towards the pure trans form of the AS layers. For this reason, we must follow a strict protocol for sample preparation during experimental repetitions. In practice, the curve of Fig. 3 was obtained by first measuring $t p_{2}$ for a long UV exposure time (in the saturation regime). Once the sample was homeotropic, we waited an additional time $t_{w}$ of the order of $30 \mathrm{~min}$ before starting a new measurement with the same sample and a smaller $t_{\mathrm{UV}}$. This protocol ensured that the value of $y$ at the beginning of each run (when we started the UV illumination) was always the same, namely,

$$
\bar{y}_{0}=y_{2} \exp \left(\frac{-t_{w}}{\tau}\right) \text {. }
$$

The above procedure was iterated using smaller values of $t_{\mathrm{UV}}$ until no plateau was observed during relaxation.

The next step consists on calculating from the kinetic Eq. (8) the value $y_{0}$ of $y$ at the end of each UV illumination. Solving this equation with $I \neq 0$ is straightforward and gives, by assuming that $\tau_{\mathrm{UV}} \ll \tau$ (an assumption that we will check a posteriori),

$$
y_{0}=1-\left(1-\bar{y}_{0}\right) \exp \left(-\frac{t_{\mathrm{UV}}}{\tau_{\mathrm{UV}}}\right)
$$

Finally, the plateau duration is given by

$$
t_{\text {plateau }}=\tau \ln \left(\frac{y_{0}}{y_{1}}\right)=\tau \ln \left[\frac{1-\left(1-y_{2} e^{-\frac{t_{w}}{\tau}}\right) e^{-\frac{t_{\mathrm{UV}}}{\tau_{\mathrm{UV}}}}}{y_{1}}\right] .
$$

This formula shows that $t_{\text {plateau }}=0$ when $t_{\mathrm{UV}}$ is smaller than

$$
t_{\mathrm{UV}}^{\min }=\tau_{\mathrm{UV}} \ln \left[\frac{1-y_{2} e^{-\frac{t_{w}}{\tau}}}{1-y_{1}}\right] \text {, }
$$

which can be estimated from the data in Fig. 3 as $t_{\mathrm{UV}}^{\min } \approx 7 \mathrm{~s}$.

Finally, this model can be used to understand the observed effect of sample aging. The simplest assumption is to suppose that, as the sample ages, a fraction $1-y_{0}$ of the azosilane molecules loses its photosensibility. In other words, the fraction $y$ of $c i s$-isomer after UV illumination is $y_{0}<1$. Under these conditions, the problem remains the same, just replacing Eq. (10) with

$$
y(t)=y_{0} \exp \left(-\frac{t}{\tau}\right)
$$

A direct consequence is that the transmittance curves remain unchanged, except that the time axis is shifted by $-\tau \ln \left(y_{0}\right)$. This agrees well with experiments shown in Fig. 5.

\section{Analysis of the experimental data}

We next proceeded to test this model with our experimental data. The unknown parameters are the characteristic time $\tau$ and the five dimensionless parameter $\alpha_{\text {trans }}, \alpha_{\text {cis }}, \beta_{\text {trans }}, \beta_{\text {cis }}$, and $\beta_{\mathrm{ES}}$. Note that $W_{a}$ does not play any role here because the director field is not distorted in the sample during the relaxation process. In practice all these parameters depend on temperature, which gives 6 free parameters for each temperature, i.e., 24 fit parameters for the 4 temperatures studied.

It is nonetheless possible to immediately reduce this number by noticing that during the sample aging the decrease of the plateau duration $t p_{1}$ is proportional to $\tau$ with a proportionality coefficient $-\ln \left(y_{0}\right)$ independent of temperature. This allowed us to directly determine the temperature dependence of $\tau$ by measuring the time shift of the curves as a function of temperature. By setting $\bar{\tau} \equiv \tau\left(-0.3{ }^{\circ} \mathrm{C}\right)$, we found that in average $\tau\left(-2^{\circ} \mathrm{C}\right) \approx 1.25 \bar{\tau}, \tau\left(-5^{\circ} \mathrm{C}\right) \approx 1.88 \bar{\tau}$, and $\tau\left(-10^{\circ} \mathrm{C}\right) \approx 3 \bar{\tau}$. This reduces to 21 the number of fit parameters.

We proceeded by simultaneously fitting the eight experimental curves of Fig. 4 with Eqs. (14) to (17) by using the Global Fit package of Igor Pro (WaveMetrics Inc.). Our fit parameters were $w t_{1}=\alpha_{\text {trans }}+2 \beta_{\text {trans }}, w c_{1}=\alpha_{\text {cis }}-2 \beta_{\text {cis }}$, $w t_{2}=\alpha_{\text {trans }}-2 \beta_{\text {trans }}, w c_{2}=\alpha_{\text {cis }}+2 \beta_{\text {cis }}, \beta_{\mathrm{ES}}$, and $\bar{\tau}$. This fit was performed by imposing to each parameter $w t_{1}, w c_{1}, w t_{2}$, $w c_{2}$, and $\beta_{\mathrm{ES}}$ to be the same for the two curves $t p_{1}(x)$ and $t p_{2}(x)$ measured at each temperature. With these constraints, we found that the global fit converged by giving $\bar{\tau}=$ $1465 \pm 161 \mathrm{~s}$ and values for the other parameters essentially independent of temperature-but with large errors. This is due to the too large number of fit parameters with respect to the number of experimental points (about 250 by taking the raw data and not the average values shown for more clarity in Fig. 4). For this reason, we redid our fit by imposing that the parameters $w t_{1}$ and $w c_{1}, w t_{2}, w c_{2}$, and $\beta_{\mathrm{ES}}$ were independent of temperature, as the first fit suggested. Under these new constraints, the number of independent fit parameters was reduced to 6 , which was much more reasonable. The values of the fit parameters obtained in this way are $\bar{\tau}=1387 \pm 82 \mathrm{~s}$, 
$\beta_{\mathrm{ES}}=810^{-5} \pm 0.057, \quad$ and $\quad w t_{1}=6.65 \pm 0.25, \quad w t_{2}=$ $5.74 \pm 0.84, w c_{1}=0.88 \pm 0.16, w c_{2}=0.89 \pm 0.12$, which gives

$$
\begin{aligned}
& \alpha_{\text {trans }}=6.2 \pm 0.11, \quad \alpha_{\text {cis }}=0.89 \pm 0.16 \\
& \beta_{\text {trans }}=0.23 \pm 0.03, \quad \beta_{\text {cis }}=0.0015 \pm 0.04
\end{aligned}
$$

This fit shows that $\beta_{\text {cis }}$ and $\beta_{\mathrm{ES}}$ are very close to 0 . This means that $W_{\mathrm{ES}}$ and $W_{\text {cis }}$ are well described by a potential of Rapini-Papoular type, proportional to $\cos (2 \theta)$. The corresponding theoretical curves are shown as solid lines in Fig. 4. As we can see, the fit is satisfactory.

With these fitted parameters, we proceeded to calculate the transmittance curves that correspond to the samples in Fig. 1. The calculations were performed with Mathematica by using Eqs. (2) and (3) and the values of the birefringence given in Fig. 2. In this calculation, angle $\theta(t)$ was numerically calculated by minimizing $W(\theta)$ at each time. The corresponding theoretical curves are shown in Fig. 6 and compare well with the experimental ones shown in Fig. 1.

Furthermore, we can reproduce the curve of Fig. 3 showing $t_{\text {plateau }}$ versus $t_{\mathrm{UV}}$. In this example, $x=0.817$ and $\delta T=$ $-0.3{ }^{\circ} \mathrm{C}$. From Eqs. (14) and (16), we calculated, using the values of the $\alpha$ and $\beta$ coefficients given above, $y_{1}=0.29$ and $y_{2}=0.192$. At this temperature, $\tau=\bar{\tau}=1387 \mathrm{~s}$. Experimentally, $t_{w} \approx 1800 \mathrm{~s}$ and $t_{\mathrm{UV}}^{\min } \approx 7 \mathrm{~s}$, which gives, by using Eq. $(23), \tau_{\mathrm{UV}} \approx 24 \mathrm{~s}$. We see here that $\tau_{\mathrm{UV}} \ll \tau$, consistently with the assumption above that led to the derivation of Eq. (21). From these values, $t_{\text {plateau }}$ can be calculated as a function of $t_{\mathrm{UV}}$ using Eq. (22). The corresponding theoretical curve, shown in Fig. 7, is again in correct agreement with the experimental data (Fig. 3).

Using the above fitted parameters, we estimated the value of $y_{0}$ for the aged sample shown in Fig. 5. For the two curves measured at $\delta T=-0.3{ }^{\circ} \mathrm{C}$, the shift is of about $150 \mathrm{~s}$, which means that $-\bar{\tau} \ln \left(y_{0}\right)=150 \mathrm{~s}$. This gives $y_{0}=0.9$ by taking $\bar{\tau}=1387 \mathrm{~s}$. This means that $10 \%$ of the azosilane molecules have lost their efficiency after two months.

Finally, we can explore the anchoring potential landscape during the planar to homeotropic transition by computing the time evolution of the anchoring potential. In Fig. 8, we plot $W[\theta(t)]$ for $\delta T=-5{ }^{\circ} \mathrm{C}$ and $x=0.817$ [same sample as in Figs. 1(b) and 6(b)]. This figure clearly shows the continuous passage between the planar and homeotropic anchoring. Another interesting point is that the potential is very flat in the transition region. This can result in the coexistence of regions with slightly different anchoring angle, which may explain why we often observe under the microscope small spatial inhomogeneities in the transmitted intensity during the transition. These local intensity variations lead to the experimental transmittance curves shown in Fig. 1 to usually pass through a maximum that is slightly smaller than 1 (contrary to the theoretical curves shown in Fig. 6).

\section{Independent measurement of the relaxation time and validation of the model}

To validate the assumptions made in the model above based on the observed evolution of LC anchoring during the isomerization of the AS layer, we performed UV-VIS spec-
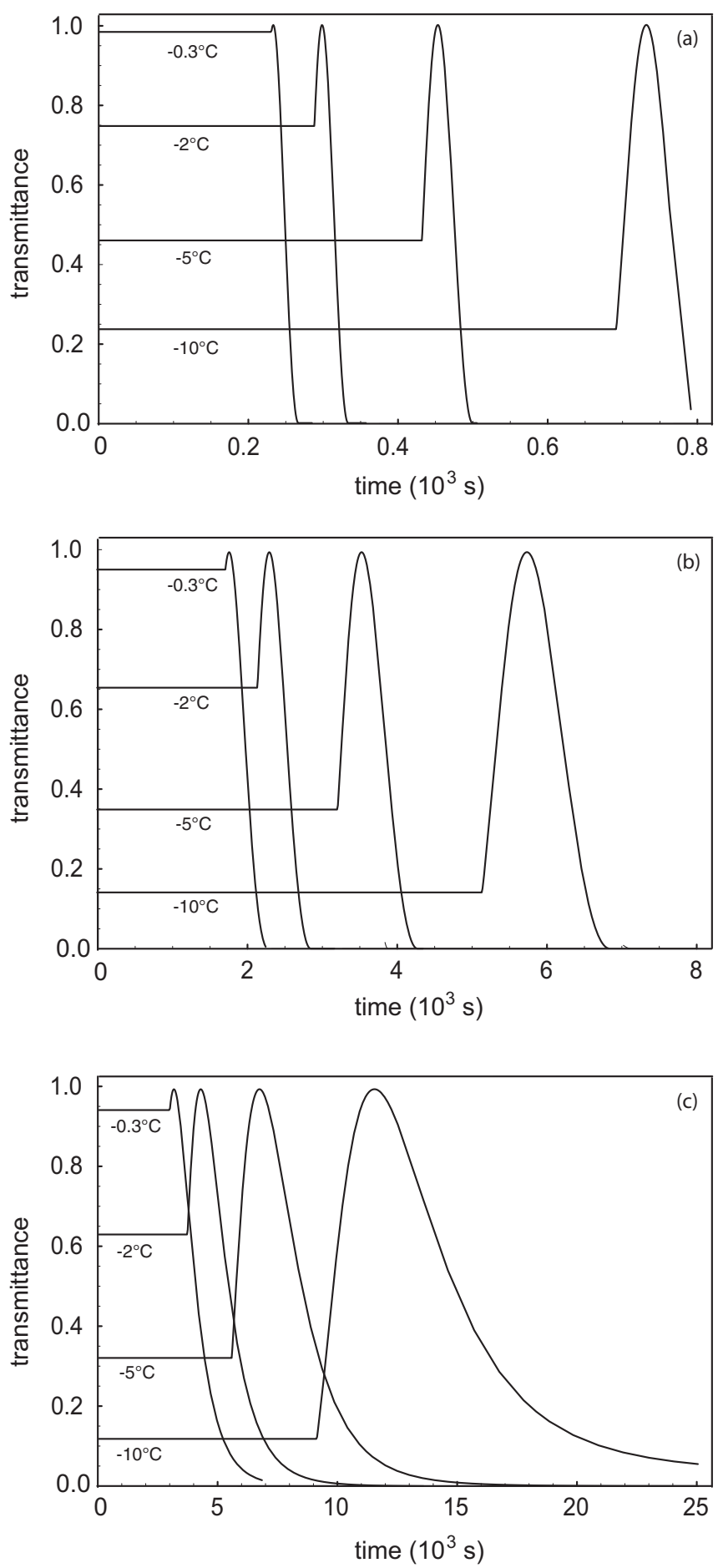

FIG. 6. Theoretical transmittance curves calculated from the model for the samples of Fig. 1 by taking $\tau=1387 \mathrm{~s}$ and the values of the parameters $\alpha$ and $\beta$ given in the text.

trophotometry measurements. We used a Shimadzu 1700 spectrophotometer equipped with a thermostatic cuvette holder, connected to a Julabo MB-12 water circulation thermostat. We built a custom adapter to study flat LC cells instead of the standard volumetric cuvettes. This double-beam instrument features a reference beam, where we placed a homeotropic LC cell. The custom holders featured identical $6 \mathrm{~mm}$ circular 


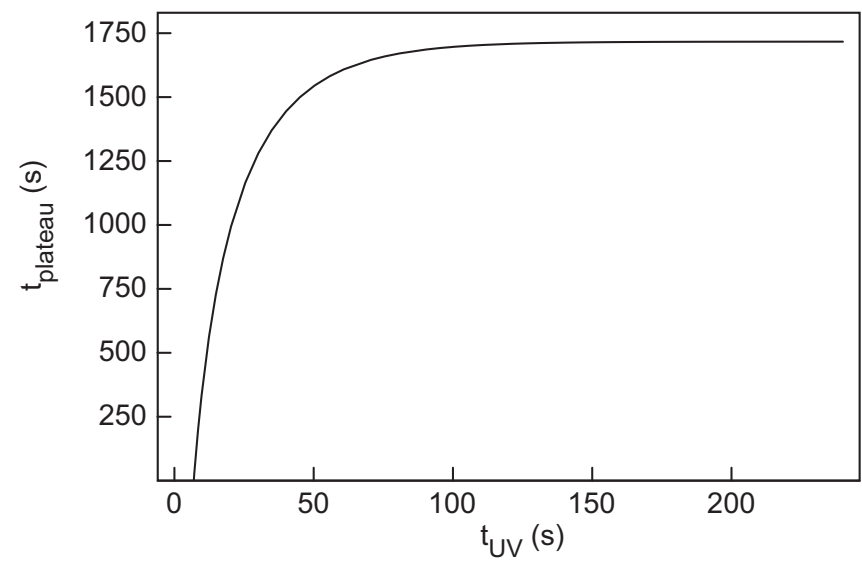

FIG. 7. Theoretical plateau duration as a function of the UV illumination time. The curve has been calculated for the same sample as in Fig. 3 and in the same experimental conditions.

windows to restrict the region of observation. Both the sample and reference cells had a gap $d=21.5 \mu \mathrm{m}$.
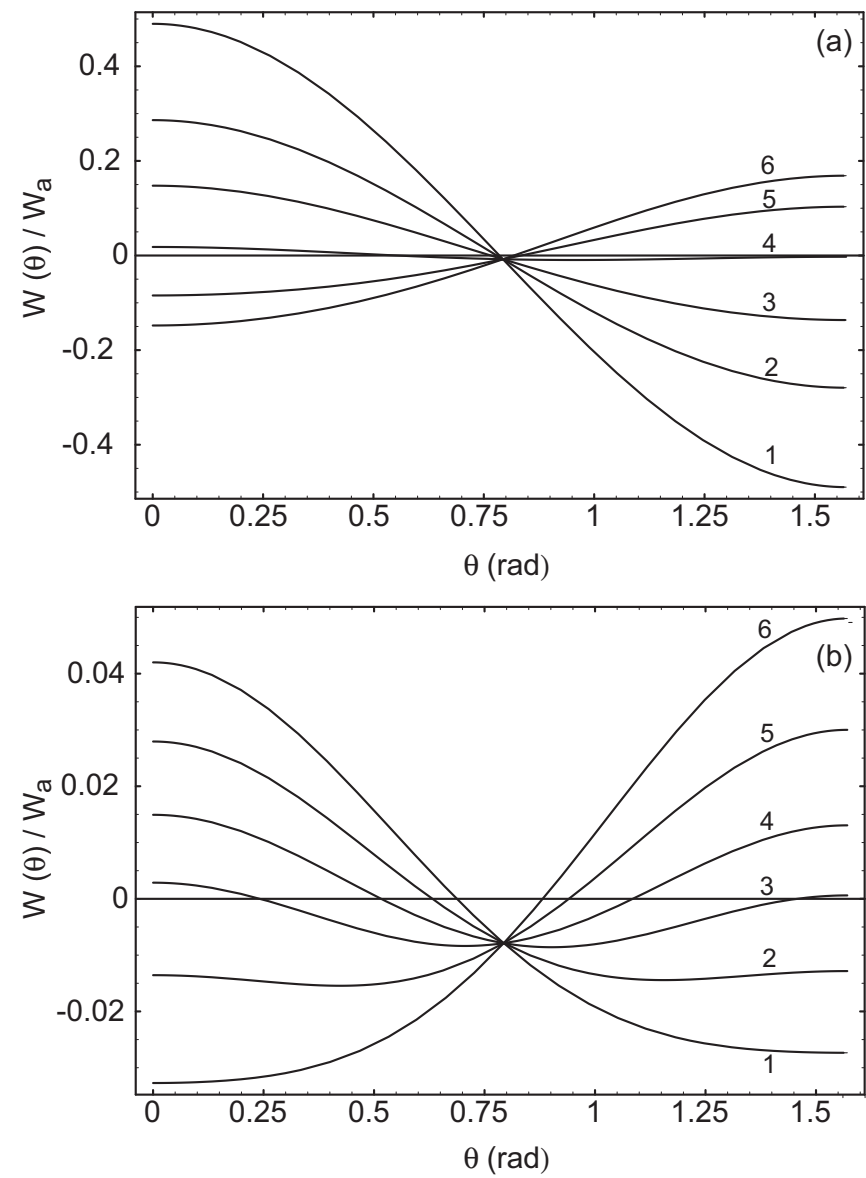

FIG. 8. Time evolution of the theoretical anchoring potential when $x=0.817$ [as in Figs. 1 (b) and 4(b)] and $\delta T=-5^{\circ} \mathrm{C}$. Graph (a) shows the global evolution of the potential. The curves 1 to 6 have been calculated at time $t=0 \mathrm{~s}, 1000 \mathrm{~s}, 2000 \mathrm{~s}, 3500 \mathrm{~s}, 6000 \mathrm{~s}$, and $\infty$, respectively. Graph (b) shows the potential during the transition from planar to homeotropic. Curves 1 to 6 have been calculated at time $t=3150 \mathrm{~s}, 3350 \mathrm{~s}, 3550 \mathrm{~s}, 3750 \mathrm{~s}, 4050 \mathrm{~s}$, and $4450 \mathrm{~s}$, respectively. In this example, $t p_{1} \approx 3200 \mathrm{~s}$ and $t p_{2} \approx 4300 \mathrm{~s}$.

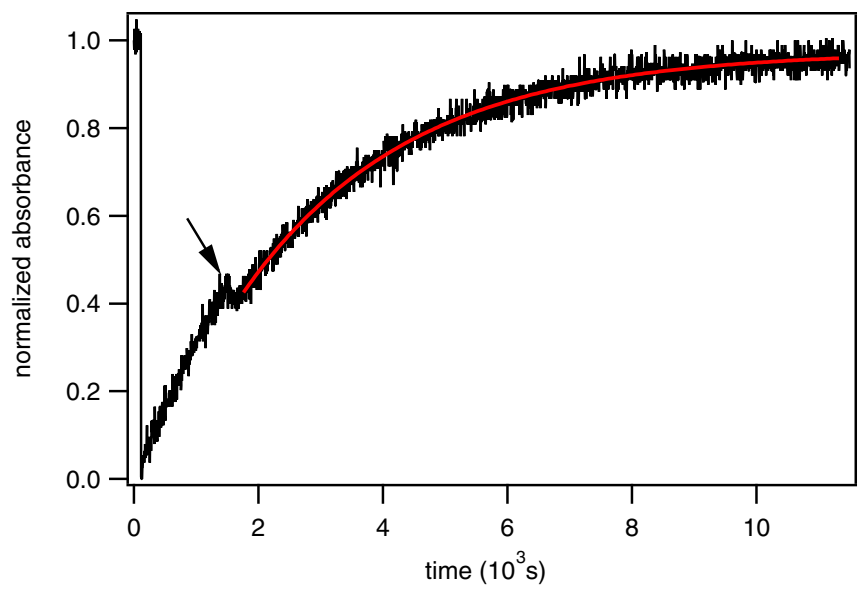

FIG. 9. Normalized absorbance of a $C_{\mathrm{ES}}=0.69$ sample at $\delta T=$ $-5^{\circ} \mathrm{C}$ after $120 \mathrm{~s}$ irradiation with UV light. The feature pointed to by an arrow corresponds to the planar-to-homeotropic transition. The solid line corresponds to a fit to first order kinetics.

We took advantage of the distinctive absorption band of the trans isomer of our azosilane compound, with a peak at $\lambda=350 \mathrm{~nm}$. At this wavelength, the cis isomer has a negligible absorbance. As a consequence, we could monitor the isomerization kinetics by using Lambert-Beers' equation, $\operatorname{Abs}_{350 \mathrm{~nm}} \propto C_{\mathrm{AS} \text { (trans). Although we lacked an abso- }}$ lute calibration to directly convert absorbance data into AS concentration, a comparison between different well-defined regions yielded useful information. All the samples used in these measurements were prepared using fused-silica plates to optimize the signal-to-noise ratio in the UV. We verified that the protocol described above for the functionalization of glass plates yielded analogous effects when applied to fused silica.

In a typical experiment, the sample was allowed to relax in the dark at the target temperature. For best results, samples were kept overnight inside the spectrophotometer, avoiding any contact with visible or UV light. This ensured that the initial condition corresponded to $100 \%$ trans AS. After ensuring that the absorbance reading at $350 \mathrm{~nm}$ was stable, we irradiated the sample with the same UV LED light source described above. Because of the configuration of the instrument, we needed to use a $45^{\circ}$ metallic mirror to irradiate the sample that was held in the thermostatic holder. After the target irradiation time, the absorbance was monitored for several hours, until the exponential relaxation had approached the high absorbance plateau, allowing to fit the relaxation to the first-order kinetics expressed in absorbances,

$$
A=A_{\infty}+\left(A_{0}-A_{\infty}\right) e^{-t / \tau} .
$$

In Fig. 9 we show a typical experiment using a sample with $C_{\mathrm{ES}}=0.69$, where irradiation with UV light was performed for $120 \mathrm{~s}$ to ensure that the AS was nearly $100 \%$ in the cis isomer. About $1500 \mathrm{~s}$ after relaxation began, we observed a shift in the absorbance curve (indicated by an arrow). By comparing with the $t p_{1}$ values measured above (Fig. 4) we concluded that this feature corresponded to the planarto-homeotropic transition, which results in a change in the sample transmittance due to the birefringence of CCN37. The duration of the transition observed here is consistent with 


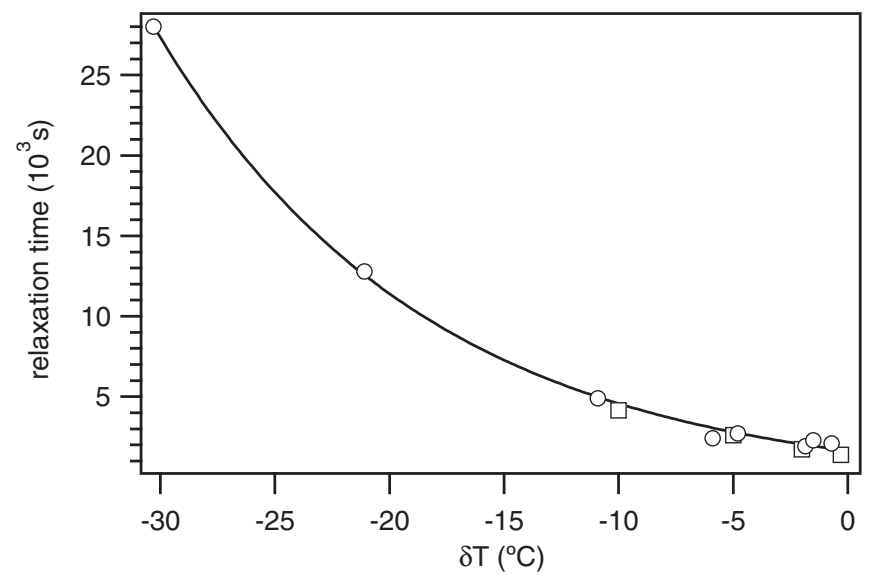

FIG. 10. Comparison between the values of the relaxation time, $\tau$, obtained from absorbance measurements $(\bigcirc)$ and from the fit to the transmittance data $(\square)$. The sample has $C_{\mathrm{ES}}=0.69$. The solid line is a guide for the eye.

$t p_{2}-t p_{1}$ for the same experimental conditions in Fig. 4. Since our reference cell was homeotropic, we only used absorbance data well after this transition was completed to obtain the relaxation time. For the data in Fig. 9, our fit yields $\tau=2711 \pm 25 \mathrm{~s}$. This value is consistent to the one obtained in Sec. III C, namely, $\tau\left(-5^{\circ} \mathrm{C}\right) \sim 1.88 \bar{\tau}=2607 \pm 154 \mathrm{~s}$. Similar measurements were performed for a wide temperature range, confirming the good agreement between values for the cis-to-trans relaxation time estimated from absorbance measurements and from the fit to the transmittance data (Fig. 10). On top of that, this result validates our assumptions on the aging of the photosensitive monolayers from which we deduced the temperature dependence $\tau(T) / \bar{\tau}$.

The absorbance data in Fig. 9 can be used to estimate the monolayer composition required to have homeotropic anchoring. If, after the $120 \mathrm{~s}$ irradiation, all AS molecules are in the cis form (consistently with the assumption in the model), and, when the plateau is finally reached, all AS molecules are in the trans form, we conclude, by comparing relative absorbances, that for anchoring to be homeotropic we need about $42 \%$ of the AS molecules to be in the trans form. Considering that this sample contains about $31 \%$ AS molecules and $69 \%$ ES molecules $\left(C_{\mathrm{ES}}=0.69\right)$, then homeotropic anchoring requires about $13 \%$ of the total silane molecules in the monolayer to be AS trans. This is consistent with the fact that $t p_{1}$ diverges when more than $84 \%$ of the silane molecules are ES (Fig. 5). In other words, the sample will be planar (cannot be homeotropic) when more than $84 \%$ of the silane molecules are ES.

\section{ZENITHAL ANCHORING ENERGY}

\section{A. Estimation from wall analysis}

From the previous transmittance measurements, it is impossible to obtain the anchoring energy $W_{a}$. However, useful information about $W_{a}$ can be obtained by examining the domain walls that form in the samples during the UV illumination. Indeed, two types of domain may develop in the samples during the homeotropic-to-planar transition

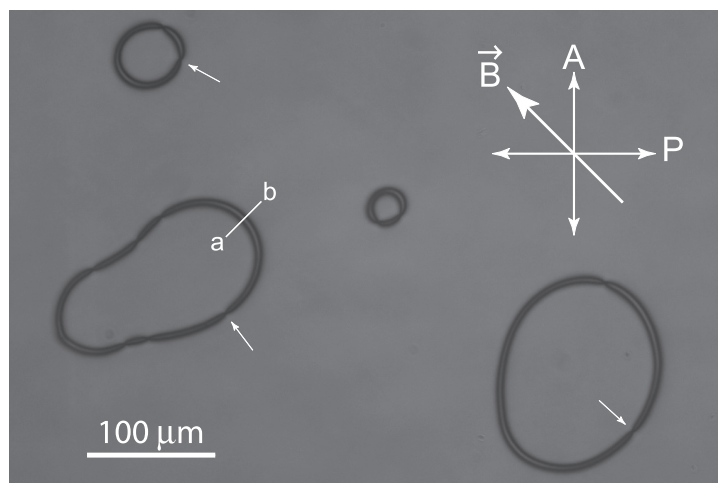

FIG. 11. Ising walls and disinclination lines (indicated by the arrows) observed immediately after the UV illumination. $d=$ $21.69 \mu \mathrm{m}$ and $C_{\mathrm{ES}}=0.69$ and $\delta T=-0.3{ }^{\circ} \mathrm{C}$.

because the director can indifferently rotate clockwise or counterclockwise in the plane perpendicular to the magnetic field. As a result, $\pi$-walls must form between these domains as shown in Fig. 11, in an image taken immediately after the UV illumination. Observation between crossed polarizers at $45^{\circ}$ of the magnetic field shows that the intensity signal measured along a line perpendicular to the wall passes through two minima (Fig. 12). This indicates that the director goes out of the plane perpendicular to the magnetic field. For this reason, the walls are Bloch walls [2]. This observation also explains the formation of the disclination lines (marked by an arrow in Fig. 11) that form on the walls. These lines separate two wall portions in which the director rotates in opposite directions. The observation of Bloch walls (rather than Ising walls in which the director would rotate in the plane perpendicular to $\vec{B}$ ) clearly indicates that the director prefers to remain parallel to the surfaces rather than perpendicular to the magnetic field. This is the result of a competition between the anchoring and the magnetic field.

To quantify this competition, we developed a very simple model for $\pi$-walls under a magnetic field. In this model, the

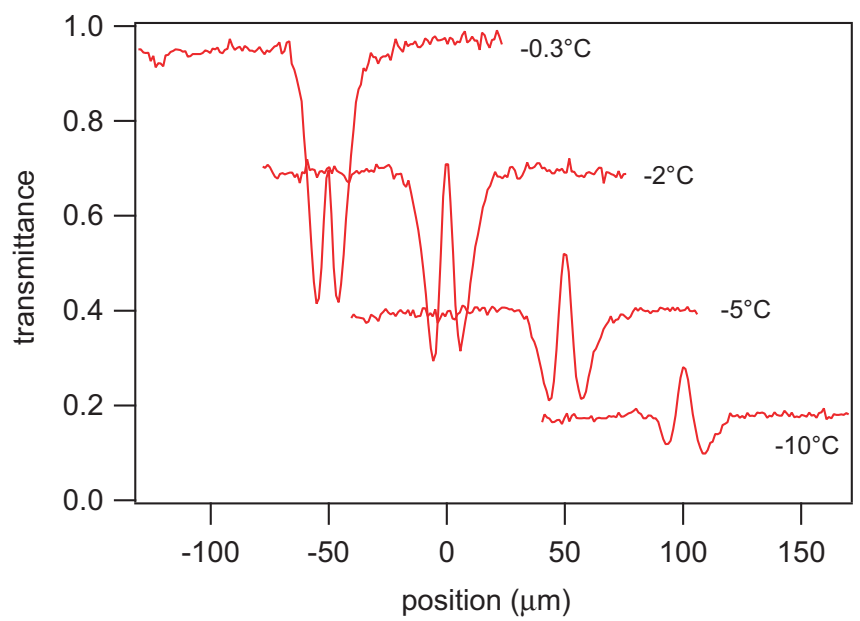

FIG. 12. Typical intensity profiles measured at different $\delta T$ between crossed polarizers along a line perpendicular to the wall and perpendicular to the magnetic field (line ab in Fig. 11). Sample thickness $d=21.69 \mu \mathrm{m}$ and $C_{\mathrm{ES}}=0.69$. 

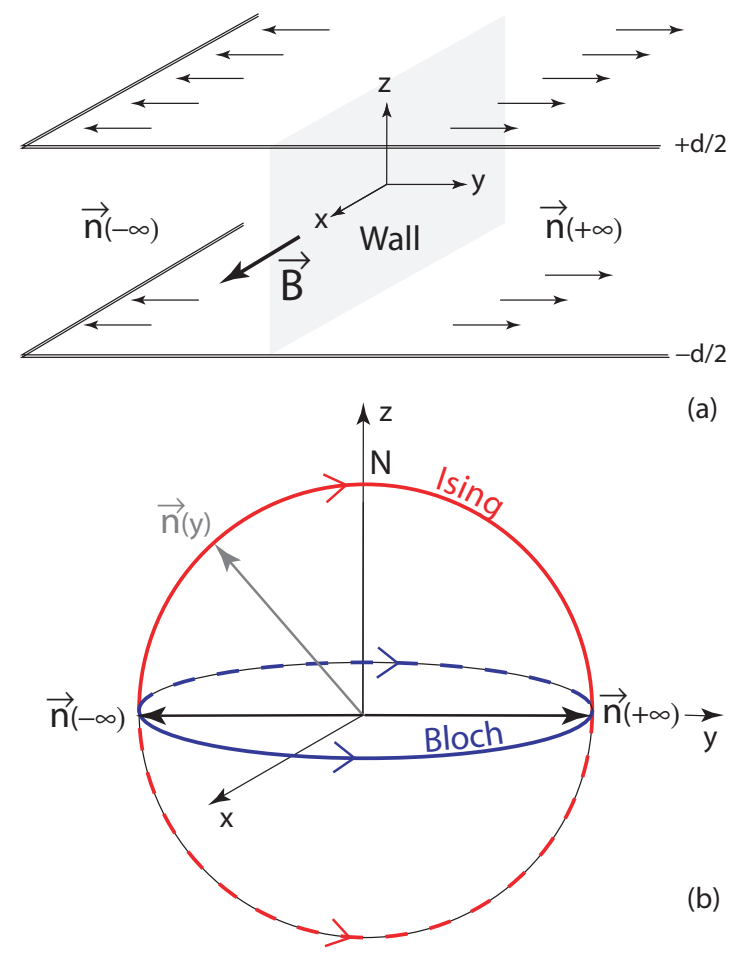

(a)

(b)

FIG. 13. (a) Coordinate system for a $\pi$-wall in a magnetic field (b) Representation on the unit sphere $S_{2}$ of the director field across a Bloch wall (blue trajectories) and an Ising wall (red trajectories). For each type of walls, there are two possible trajectories (drawn in solid and dashed lines, respectively) depending on the sense of rotation of the director.

director $\vec{n}$ has for components $n_{x}, n_{y}$, and $n_{z}=\left(1-n_{x}^{2}-\right.$ $\left.n_{y}^{2}\right)^{1 / 2}$. The $z$ axis is taken perpendicular to the surfaces, the magnetic field $\vec{B}$ is parallel to the $x$ axis, and the wall is perpendicular to the $y$ axis [Fig. 13(a)]. We need to minimize the total energy including the elastic, magnetic, and anchoring energies with $n_{x}(y, z)$ and $n_{y}(y, z)$ and the boundary conditions:

$$
\begin{aligned}
& n_{y}(+\infty, z)=1, \quad n_{x}(+\infty, z)=n_{z}(+\infty, z)=0, \\
& n_{y}(-\infty, z)=-1, \quad n_{x}(+\infty, z)=n_{z}(+\infty, z)=0 .
\end{aligned}
$$

This is a very difficult task which cannot be performed analytically, even in isotropic elasticity. For this reason and to determine for which typical value of the anchoring energy ones passes from an Ising to a Bloch wall, we assume that $n_{x}$ and $n_{y}$ do not depend on $z$ and we propose as an ansatz that

$$
\begin{aligned}
& n_{x}(y)=\frac{C}{\cosh \left(\frac{y}{\xi}\right)}, \\
& n_{y}(y)=\tanh \left(\frac{y}{\xi}\right),
\end{aligned}
$$

where $4 \xi$ is the typical wall thickness. This director field satisfies the boundary conditions Eq. (26) and describes an Ising wall when $C=0$ and a Bloch wall when $C=1$. In the first case, the director describes one of the two red trajectories passing though the poles on the unit sphere [Fig. 13(b)]. In the second case, the director describes on the unit sphere one of the two blue trajectories on the equator [Fig. 13(b)]. In the other cases $(0<C<1)$, the director rotates in an intermediate plane.

To calculate in which limit a Bloch wall is observed, we calculated the total energy of the wall. Assuming $K=K_{1}=$ $K_{3}$ (the wall is here of splay-bend type) the Frank and magnetic energies reduce to

$$
\frac{f_{v}}{K}=\frac{1}{2}\left(n_{x, z}^{2}+n_{y, y}^{2}+n_{z, y}^{2}\right)+\frac{1}{2} \frac{n_{x}^{2}}{\xi_{m}^{2}},
$$

where $\xi_{m}=\sqrt{\frac{\mu_{0} K}{-\chi_{a}}} \frac{1}{B}$ is the magnetic coherence length, $\mu_{0}$ is the vacuum permeability, and $\chi_{a}$ is the (negative) magnetic anisotropy. For the surface energy, we chose a simplified form that neglects the $\cos (4 \theta)$-term (this is justified as $\beta \ll \alpha$ for ES and AS). In this case, the surface energy (which we take equal to 0 at infinity in the $y$ direction) reads at time $t=0$ :

$$
\frac{f_{s}}{K}=\frac{\alpha(0)}{2 l_{a}}[\cos (2 \theta)+1]=\frac{\alpha}{l_{a}}\left[1-n_{x}(y)^{2}-n_{y}(y)^{2}\right],
$$

where $l_{a}=\frac{K}{W_{a}}$ is the anchoring extrapolation length and $\alpha \equiv$ $\alpha(0)=C_{\mathrm{ES}}+\alpha_{\mathrm{cis}}\left(1-C_{\mathrm{ES}}\right)$.

The next step is to calculate the wall energy $F$. A straightforward integration of $f_{v}$ and $f_{s}$ over $y$ and $z$ coordinates yields (per unit length along $x$ )

$$
\frac{F}{K}=\frac{d}{\xi}+\xi\left(\frac{4 \alpha}{l_{a}}-4 \frac{\alpha C^{2}}{l_{a}}+\frac{C^{2} d}{\xi_{m}^{2}}\right) .
$$

Minimization as a function of $\xi$ gives

$$
\xi^{-1}=\sqrt{\frac{4 \alpha\left(1-C^{2}\right)}{l_{a} d}+\frac{C 2}{\xi_{m}^{2}}}
$$

and

$$
\frac{F}{K}=\sqrt{\frac{4 \alpha}{l_{a}}+C^{2}\left(\frac{d}{\xi_{m}^{2}}-\frac{4 \alpha}{l_{a}}\right)} .
$$

From these formulas we deduce that if $l_{a}>\frac{4 \alpha \xi_{m}^{2}}{d}$ the wall is of the Ising type $(C=0)$, of width $4 \xi=2 \sqrt{l_{a} d / \alpha}$, whereas if $l_{a}<\frac{4 \alpha \xi_{m}^{2}}{d}$ a Bloch wall of width $4 \xi=4 \xi_{m}$ must be observed $(C=1)$. Experimentally, we are clearly in the second case, which means that

$$
l_{a}=\frac{K}{W_{a}}<\frac{4 \alpha \xi_{m}^{2}}{d} .
$$

In $\mathrm{CCN}-37, \xi_{m} \sim 5 \mu \mathrm{m}$ by taking $B=1 \mathrm{~T}$ and the values of $\chi_{a}$ and $\mathrm{K}$ (with $K \approx \frac{K_{1}+K_{3}}{2}$ ) given in Ref. [18]. As a consequence this very simple model predicts a wall thickness of the order of $4 \xi_{m} \approx 20 \mu \mathrm{m}$, which agrees with the intensity profiles measured experimentally (see Fig. 12) and an extrapolation length $K / W_{a}<5 \mu \mathrm{m}$ (knowing that for the sample shown in Fig. $11, \alpha \approx 1)$. In practice, $l_{a}$ could be deduced by numerically calculating the director field and the corresponding transmittance profiles and by then comparing the theoretical profiles with the experimental ones. However, these calculations are complicated and may not be precise if the anchoring length is too small. For this reason, we preferred using another more simple method. 

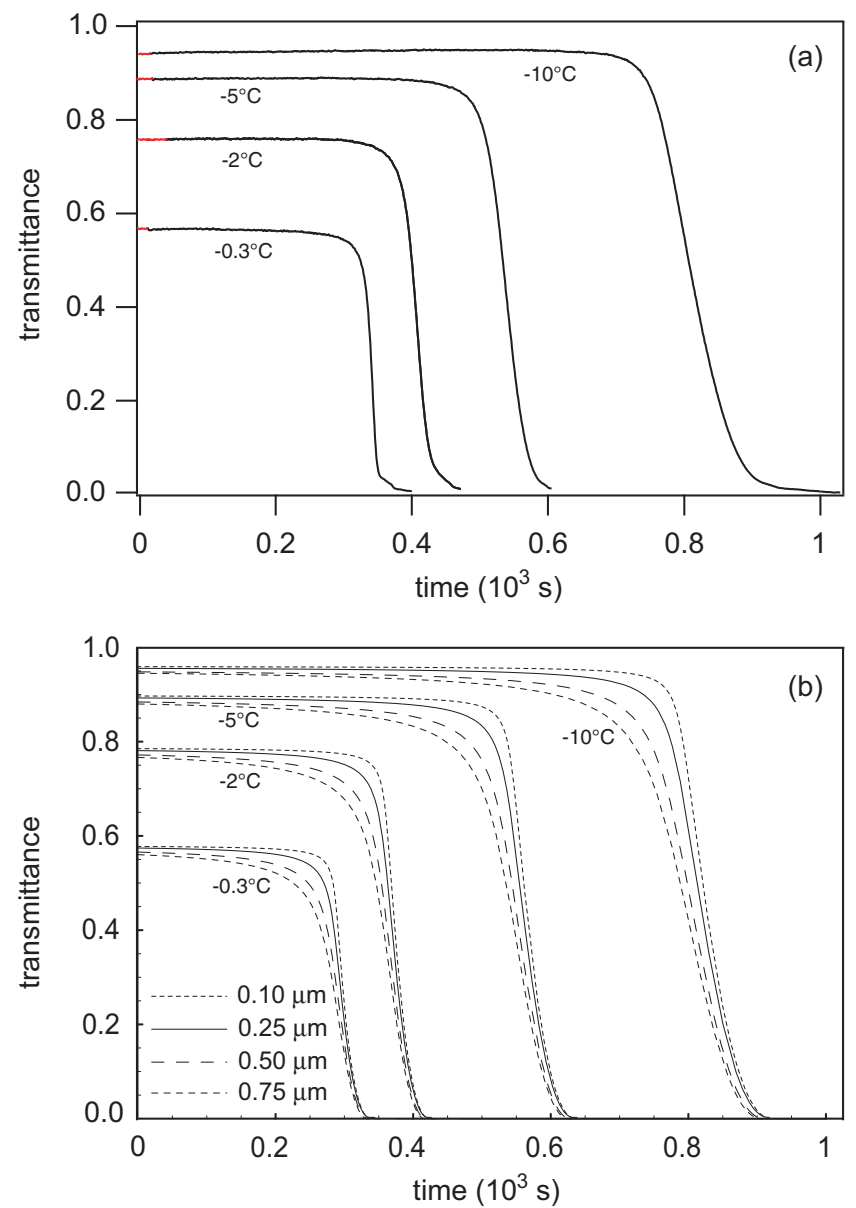

FIG. 14. Transmittance curves in a mixed sample. (a) Experimental curves measured in a sample of thickness $d=21.5 \mu \mathrm{m}$ and $C_{\mathrm{ES}}=$ 0.356. (b) Corresponding theoretical curves calculated for different values of $l_{a}$ (given on the graph) by taken the values of the parameters $\alpha, \beta$, and $\tau$ given in Sec. III C, and the values of the birefringence given in Fig. 2.

\section{B. Estimation from cells with hybrid anchoring}

Another way for estimating $l_{a}$ consists of preparing hybrid samples, with one surface treated with the photosensible monolayer while the other is treated for strong homeotropic anchoring. In this case, the director field is not homogeneous inside the sample. When the anchoring energy on the photosensitive plate becomes small [i.e., close to the end of the plateau, see Fig. 8(b)] the transmittance curves of Fig. 14(a) reveal round off at the end of the plateau, before they fall to 0 . This is due to nematic elasticity, which brings the anchoring angle on the photosensitive plate closer to the orientation of the strong homeotropic anchoring on the complementary plate. To quantify this effect, we calculated the expected transmittance curves in this geometry. Let $\theta(z)$ be the angle between the director and the normal to the surfaces. Assuming isotropic elasticity $\left(K=\frac{K_{1}+K_{3}}{2}\right.$ ), it can be easily shown [21] that this angle varies linearly from 0 on the homeotropic plate to $\theta_{d}=\theta(d)$ on the photosensible plate:

$$
\theta(z)=\theta_{d} \frac{z}{d}
$$

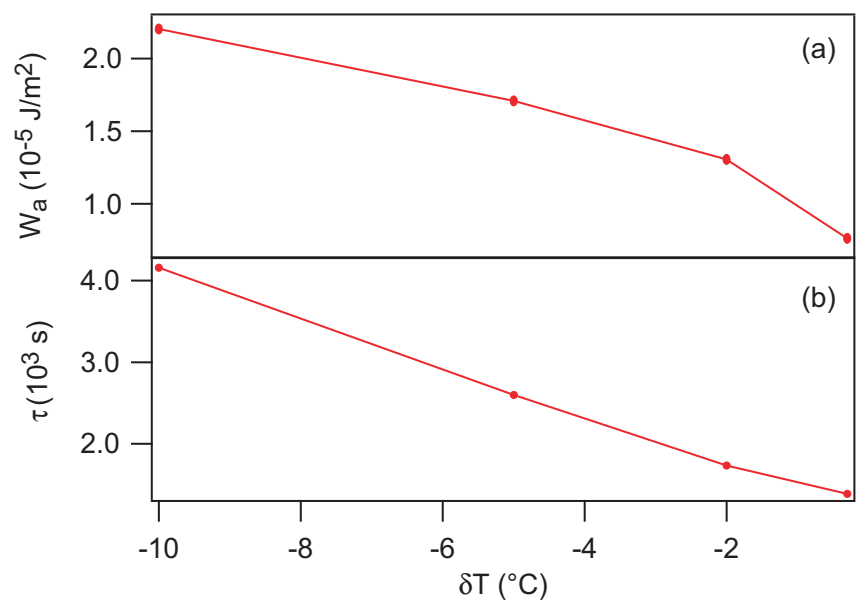

FIG. 15. Order of magnitude of the anchoring energy $W_{a}$ (a) and of the characteristic time $\tau$ (b) as a function of temperature.

At each time, and for a given value of $l_{a}$, the anchoring angle $\theta_{d}$ was obtained numerically by minimizing the total energy of the sample

$$
\frac{F_{\text {total }}}{W_{a}}=\frac{W\left(\theta_{d}, t\right)}{W_{a}}+\frac{1}{2} l_{a} \frac{\theta_{d}^{2}}{d},
$$

with the values of the parameters $\alpha, \beta$, and $\tau$ given in Sec. III C. The transmittance was then numerically calculated using Eq. (2) with

$$
\Phi=\frac{2 \pi \Delta n}{\lambda} \int_{0}^{d} \sin ^{2}\left(\frac{\theta_{d} z}{d}\right) d z=\frac{\pi d \Delta n}{\lambda}\left(1-\frac{\sin 2 \theta_{d}}{2 \theta_{d}}\right),
$$

and using the values of $\Delta n$ given in Fig. 2. The computed transmittance curves [Fig. 15(b)] are consistent with the experimental ones if $l_{a} \sim 0.1-0.3 \mu \mathrm{m}$, although these measurements are not precise enough to resolve the temperature dependence of $l_{a}$. This order of magnitude is compatible with our observations on the Bloch walls, which showed that $l_{a}<5 \mu \mathrm{m}$. Using $l_{a} \sim 0.2 \mu \mathrm{m}$ and the values of $K_{1}$ and $K_{3}$ from the literature [18], we estimated $W_{a}$ as a function of temperature (see Fig. 15 in which we also reported $\tau$ as a function of temperature).

\section{AZIMUTHAL ANCHORING ENERGY AND MEMORIZATION}

\section{A. Evidence of anchoring memory}

In this section, we investigate whether an easy anchoring direction is memorized during the UV illumination of the sample under a static magnetic field. To address this issue, we prepared a photosensitive sample of thickness $d=49.96 \mu \mathrm{m}$ (the choice of this larger thickness will be justified later), where both surfaces were treated with the same silane solution, with $C_{\mathrm{ES}}=0.69$. All the measurements were performed between crossed polarizers, with the polarizer (respectively, analyzer) parallel to the $x$ (respectively, $y$ ) axis (Fig. 16). Once the temperature was stabilized, the sample was illuminated with the UV light until the planar anchoring was reached. During this operation the magnetic field was fixed and parallel to the analyzer (along $y$ direction). In this way, the director rotated in 


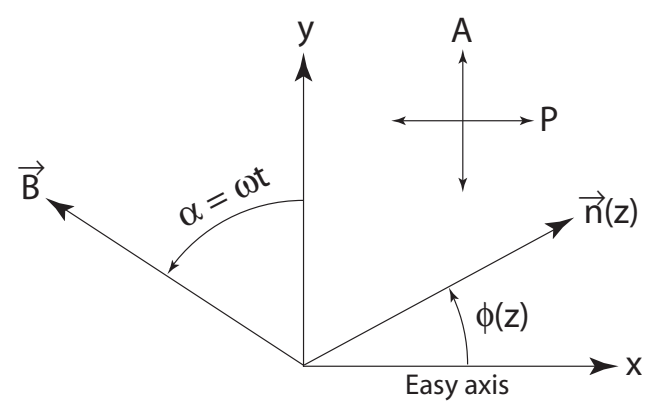

FIG. 16. Definition of the coordinate system and angles $\alpha$ and $\phi(z)$ used in Sec. V A.

the vertical $(x, z)$ plane and the final photostationary state was a planar sample oriented along the $x$ direction. The illumination time was chosen long enough to be in the saturation regime at the end of the UV exposure (Fig. 3). To determine whether the $x$ direction imposed by the magnetic field had become an easy anchoring direction, we slowly turned down the magnetic field and observed the sample under the microscope. Two regimes were qualitatively observed depending on temperature:

(i) - a sliding anchoring regime close to the transition temperature $\left(-3{ }^{\circ} \mathrm{C}<\delta T<0{ }^{\circ} \mathrm{C}\right)$ in which the director rotated continuously, but irregularly, with the magnetic field;

(ii) - a stick-slip-like regime (typically when $\delta T<$ $-3{ }^{\circ} \mathrm{C}$ ), in which the director rotated by successive jumps by nucleation and propagation of anchoring walls on the surfaces.

These observations clearly suggest that the sample memorizes an easy anchoring direction during the UV illumination with an azimuthal anchoring energy that strongly depends on the temperature. In the next two subsections we show how we measured the azimuthal anchoring energy, $\bar{W}_{a}$ (or, equivalently, the extrapolation length $\bar{l}_{a}=K_{2} / \bar{W}_{a}$ ), as a function of temperature. Finally, we will look at the problem of the memorization of the easy axis once the sample has returned in its homeotropic state.

\section{B. Azimuthal anchoring energy in the sliding regime}

Close to the clearing transition, the azimuthal anchoring will be in the weak (sliding) regime, so we can apply the same method as in Ref. [22] to measure the azimuthal anchoring energy. In short, we measured the sample transmittance between crossed polarizer and analyzer as a function of angle $\varphi=\omega t$ between the rotating magnetic field and the $y$ axis. In all experiments, the angular velocity was $\omega=0.052 \mathrm{rad} / \mathrm{s}$, for which viscosity effects are seen to be negligible. Figure 17(a) shows a typical curve measured at $\delta T=-2.3^{\circ} \mathrm{C}$. The transmittance profile is distorted with respect to the pure sine function, $\sin (4 \omega t)$, which is expected under a not perfect sliding planar anchoring. More precisely, the transmittance function has a period $\alpha_{1}+\alpha_{2}=\pi$ (instead of $\pi / 2$ ), where $\alpha_{1}$ and $\alpha_{2}$ are the neighboring angular intervals between three successive maxima, as shown in Fig. 17(a). Here, $\alpha_{1}$ (respectively, $\alpha_{2}$ ) characterizes the time the director spends along the $x$ (respectively, $y$ ) direction. We observe that $\alpha_{1}$ is always larger than $\alpha_{2}$, which means that the $x$
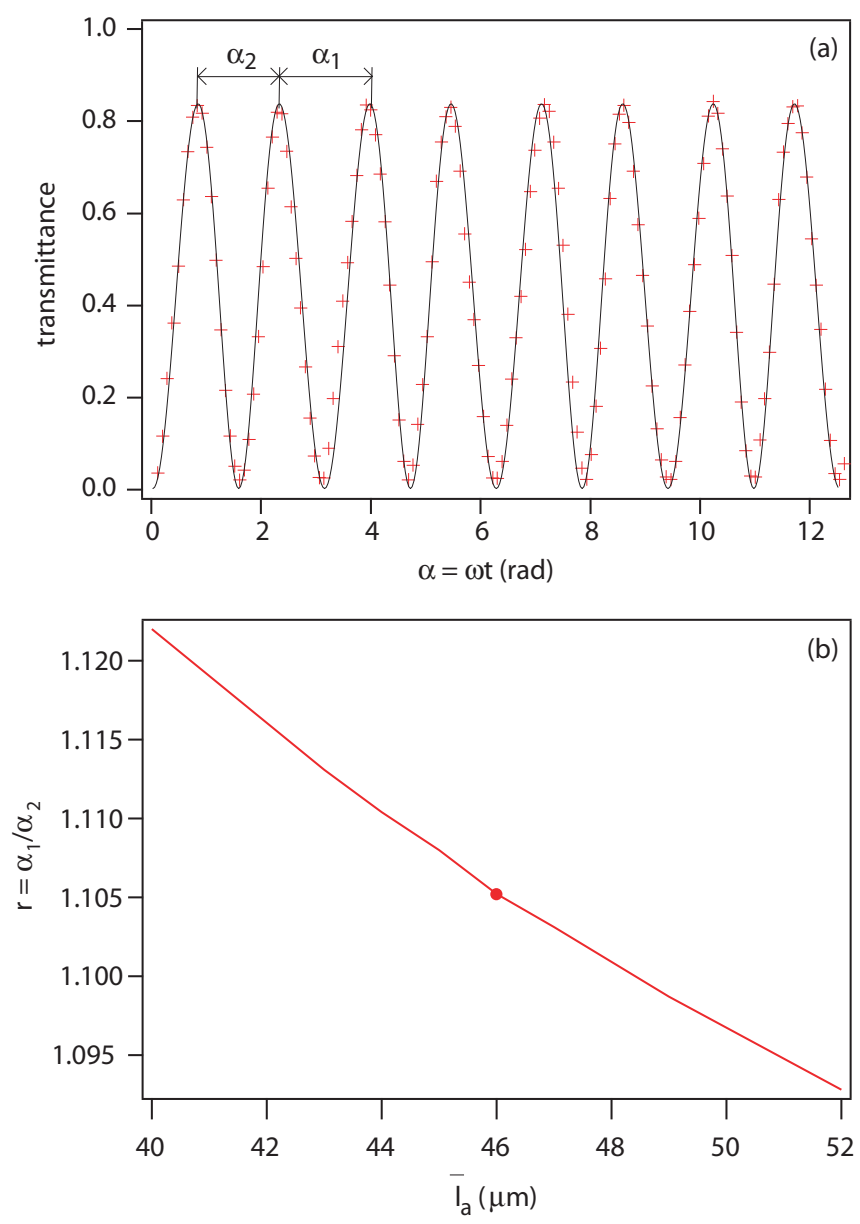

FIG. 17. (a) Transmittance between crossed polarizer and analyzer as a function of the rotation angle of the magnetic field for $d=49.96 \mu \mathrm{m}$ and $\delta T=-2.1{ }^{\circ} \mathrm{C}$. The solid line is the theoretical curve that corresponds to $\bar{l}_{a}=46 \mu \mathrm{m}$. In this example $r=\alpha_{1} / \alpha_{2}=$ 1.105; (b) Computed values of the $r$-ratio as a function of $\bar{l}_{a}$. From the previous experimental value $r=1.105$ we deduced $\bar{l}_{a}=46 \mu \mathrm{m}$. $d=49.96 \mu \mathrm{m}$ and $\delta T=-2.1^{\circ} \mathrm{C}$.

direction is an easy anchoring axis along which the director tends to align. It turns out that a precise measurement of $\bar{l}_{a}$ can be directly extracted from a measurement of the ratio $r=\alpha_{1} / \alpha_{2}$.

To show this result, let us first solve the torque equations in the quasistatic limit. The latter read, in the bulk,

$$
K_{2} \frac{\partial^{2} \phi}{\partial t^{2}}+\frac{-\chi_{a}}{2 \mu_{0}} B^{2} \sin (2 \omega t-2 \phi)=0,
$$

and, on the surfaces,

$$
\begin{gathered}
K_{2} \frac{\partial \phi}{\partial z}=\bar{W}_{a} \sin (2 \phi) \text { at } z=0, \\
K_{2} \frac{\partial \phi}{\partial z}=-\bar{W}_{a} \sin (2 \phi) \text { at } z=d,
\end{gathered}
$$

where $\phi(z)$ is the angle between the director and the $x$ axis (Fig. 16). Note that we took here the azimuthal anchoring potential in the usual Rapini-Papoular form $-\frac{1}{2} \bar{W}_{a} \cos (2 \phi)$ (with $\bar{W}_{a}>0$ ). 
From the above equations, the director profile can be obtained analytically in the limit $d \gg \xi_{m}$ (which is met experimentally since $d \approx 50 \mu \mathrm{m}$ and $\xi_{m} \approx 5 \mu \mathrm{m}$ ) [22]. The solution reads, by using the dimensionless variable $Z=z / d$ :

$$
\phi(Z)=\omega t+\left(\phi_{S}-\omega t\right) \frac{\cosh \left(\frac{Z-1 / 2}{d / \xi_{m}}\right)-1}{\cosh \left(\frac{1}{2 d / \xi_{m}}\right)-1},
$$

where $\phi_{S}$ is the angle on the surfaces given by the equation

$$
\frac{\xi_{m}}{\bar{l}_{a}} \sin \left(2 \phi_{S}\right)+\sin \left(\phi_{S}-\omega t\right)=0 .
$$

We calculated the transmittance curves that corresponds to the director profile given by Eq. (40) and to $\phi_{S}$ given by Eq. (41) using Mathematica and the Jones matrix formalism. The sample thickness was discretized into 100 slabs, which was largely enough to ensure convergence. The numerical values of the material constants are given in the Appendix. The transmittance curves depend on $\bar{l}_{a}$, which is unknown and can be fitted by comparing the computed curves with the experimental ones [Fig. 17(a)]. In fact, the most remarkable feature of the transmittance curves, namely, the asymmetry between $\alpha_{1}$ and $\alpha_{2}$ depends monotonically on $\bar{l}_{a}$. In Fig. 17(b) we show the computed dependence of the ratio between these two angles, $r=\alpha_{1} / \alpha_{2}$, as a function of $\bar{l}_{a}$. By measuring $r$ in a experimental realization and comparing with this calibration curve, we can extract the value for $\bar{l}_{a}$. For instance, for the data set in Fig. 17(a) we measured $r=1.105$, which corresponds to $\bar{l}_{a}=46 \mu \mathrm{m}$ in Fig. 17(b). The computed curve agrees very well with the experimental one. This checking is important because it confirms that the anchoring remains planar during a complete revolution, an assumption that we made implicitly from the beginning.

This method was systematically applied in the sliding regime, for temperatures close to the clearing transition temperature. The values of $\bar{l}_{a}$ obtained this way and the corresponding values of the anchoring energy $\bar{W}_{a}$ calculated with the values of $K_{2}$ given in the Appendix are shown in Fig. 18. This graph shows that $\bar{l}_{a}\left(\bar{W}_{a}\right)$ strongly increases (decreases) but does not diverge (vanish) when the transition temperature is approached. This means that even at the transition temperature an easy anchoring direction is memorized.

\section{Azimuthal anchoring energy far from the transition}

The method used in the previous section to estimate $\bar{l}_{a}$ becomes imprecise when the latter is too small, typically less than $20 \mu \mathrm{m}$, and fails to apply if $\bar{l}_{a}<2 \xi_{m} \approx 12 \mu \mathrm{m}$, since $\phi_{S}$ ceases to be a monotonically increasing function of $\omega t$ and presents jumps for some values of $\omega t$ with an hysteretic behavior when the direction of rotation is reversed. In this regime, it is still possible to estimate $\bar{W}_{a}$ for magnetic field rotations smaller than $\pi / 4$ by calculating the theoretical transmittance curves with the Jones matrix method and the director field profile obtained from the numerical solution of Eqs. (37)-(39). The calculations were performed with Mathematica by using an appropriate shooting method to solve the differential equations and selecting the good solution, looking for the value of $\bar{l}_{a}$ that best fits the experimental
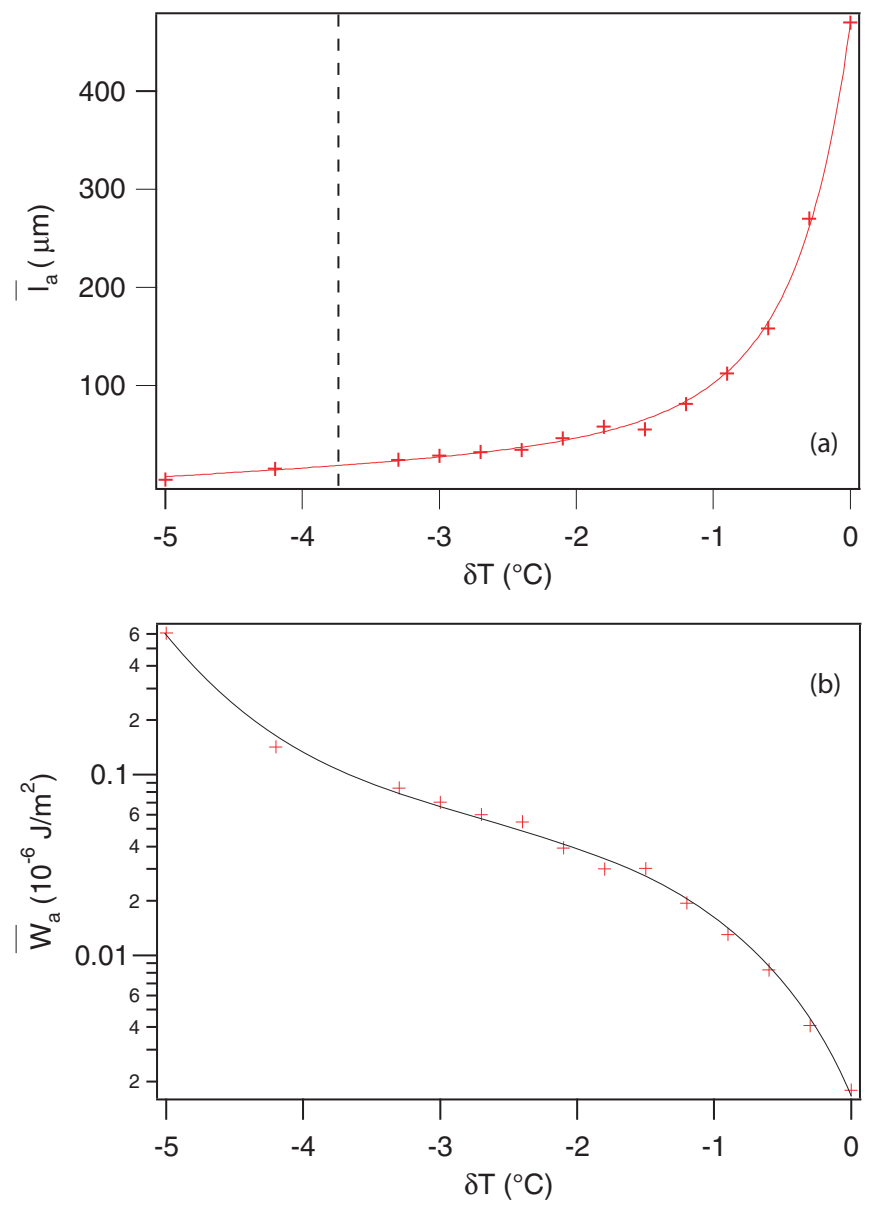

FIG. 18. (a) Azimuthal extrapolation length as a function of temperature. The points on the right of the vertical dashed line have been obtained with the rotating-field method. The points on the left have been measured with the static method. (b) Azimuthal anchoring energy as a function of temperature. The solid lines are guides for the eye.

data. An example is shown in Fig. 19 for $\delta T=-5^{\circ} \mathrm{C}$. Three theoretical curves, calculated by taking the values of the material constants given in the Appendix, are compared to the data. We observe that this method yields an estimate for $\bar{l}_{a}$ with an accuracy better than $1 \mu \mathrm{m}$. The values of $\bar{l}_{a}$ obtained this way are combined in Fig. 18 with those in the sliding anchoring regime. By extrapolating this curve, one sees that when $\delta T<-6^{\circ} \mathrm{C}, \bar{l}_{a} \leqslant 1 \mu \mathrm{m}$. Unfortunately, we were not able to measure $\bar{l}_{a}$ in this regime of strong anchoring. We observe that $\bar{W}_{a} \sim 10^{-6} \mathrm{~J} \mathrm{~m}^{-2}$ as we enter this regime, consistently with estimates reported in the literature for a shorter chain azobenzene derivative [15].

\section{About the memorization of the easy axis}

A final question we address is whether the easy axis memorized in planar samples within the strong anchoring regime can be reset and reconfigured. As is the usual case under strong planar anchoring, the memory of an easy axis is not lost even if the sample is cycled through the isotropic phase. In our case, however, we have an additional resource: we can take advantage of the reversible isomerization that returns the 


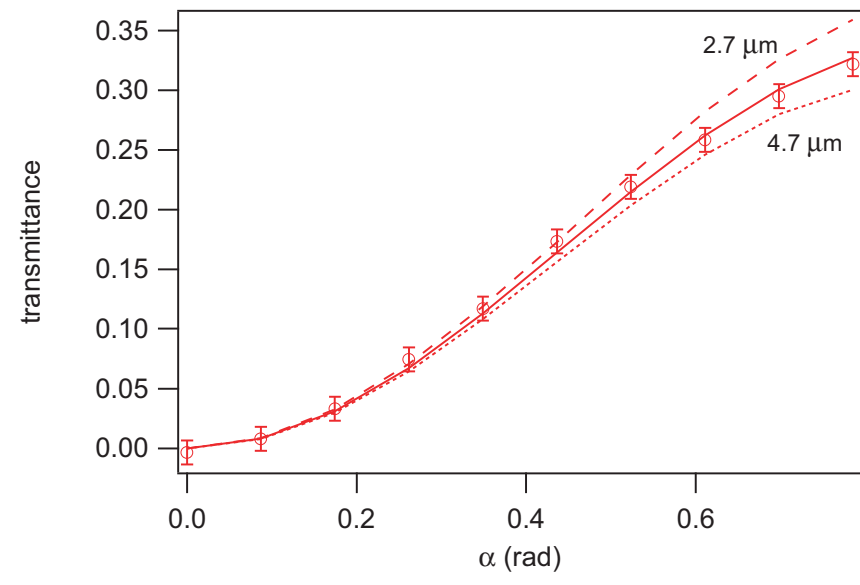

FIG. 19. Transmittance as a function of the rotation angle of the magnetic field at $\delta T=-5^{\circ} \mathrm{C}$. The crosses are the experimental points. The solid line has been calculated by taking $\bar{l}_{a}=3.7 \mu \mathrm{m}$. The dashed and dotted lines correspond to $\bar{l}_{a}=2.7$ and $4.7 \mu \mathrm{m}$, respectively.

LC sample into the homeotropic alignment when the AS layer relaxes to the trans form. To address this issue, we used a fresh sample and worked at $\delta T=-10^{\circ} \mathrm{C}$, well into the strong azimuthal anchoring regime. In a first step, we proceeded to irradiate the sample with UV light for $5 \mathrm{~min}$ in the presence of a $1 \mathrm{~T}$ magnetic field oriented at $45^{\circ}$ from the crossed polarizer and analyzer. As expected, the sample became planar, oriented perpendicularly to the magnetic field. We next allowed the sample to relax until transmittance vanished, indicating that the LC cell had returned to homeotropic. In a second step, we proceeded to irradiate the sample with UV again, but this time with the magnetic field parallel to the polarizer, which should result in a planar sample with the director parallel to the analyzer and, thus, with vanishing transmittance. Nevertheless, we measured a transmittance of around 0.15 , which is an unambiguous indication that the director escaped from the alignment plane imposed by the magnetic field, presumably forced by the anchoring plates, which remember the easy axis at $45^{\circ}$ imprinted in the previous step. Let us recall (Sec. III B) that, even after the LC cell had relaxed to homeotropic alignment at the end of the first step, a significant fraction of $c i s$ isomers would remain that, we conjecture, contribute to preserve the memory of the initial alignment step.

To test this hypothesis, we performed a third step with the same sample, consisting in returning all AS molecules into the trans form. We did this by forcing the cis-to-trans conversion with strong visible light, for which we used the lamp of the microscope, without polarizer and red filter, during $15 \mathrm{~min}$. Then we repeated the second step above, namely irradiating with UV light with the magnetic field parallel to the polarizer. This resulted in a planar sample with vanishing transmittance, confirming that the return of the AS layer to the trans isomer had effectively erased all memory from previous steps.

\section{CONCLUSION}

In this paper, we have performed a quantitative study of the photoinduced anchoring transition in nematic liquid crystal cells bound by self-assembled monolayers prepared with a mixture of photosensitive and nonphotosensitive silanes. We have presented a systematic set of experiments of the relaxation kinetics of the cis-to-trans isomerization, which correlates with a change from planar to homeotropic alignment of the director field, as a function of temperature and monolayer composition. Using simple photochemistry kinetics combined with well-established arguments for LC anchoring, we have proposed a model that enables to relate the observed intrinsic relaxation times with material and surface anchoring parameters. We have measured the zenithal and the azimuthal anchoring energies under different experimental conditions and we have revealed an interesting feedback mechanism during the photoinduced planar alignment of the samples. This mechanism leads to the memorization of a planar easy axis direction, which can be erased and reconfigured by forcing the photosensitive layer into the trans isomeric form. Besides providing with a model system where to study reversible anchoring transitions, our work paves the way for the development of photosensitive LC devices where the relaxation time can be finely tuned by means of the monolayer composition, and a memory of the planar anchoring direction can be either maintained or erased depending on sample processing history.

\section{ACKNOWLEDGMENTS}

P.O. thanks Guilhem Poy for useful discussions and his help for writing the program in Mathematica with the Jones matrices. J.I.-M. thanks J. M. Pagès for his help during the initial assessment of the surface silanization protocol and acknowledges funding from MINECO (Project No. FIS201678507-C2-1-P) and the hospitality from ENS Lyon.

\section{APPENDIX: MATERIAL CONSTANTS}

In our calculations we needed the values of the birefringence $\Delta n$, the twist constant $K_{2}$, and the magnetic coherence length $\xi_{m}$. The birefringence was measured following the protocol detailed in Ref. [18] but using red light $(\lambda=633 \mathrm{~nm})$ (Fig. 20). The values of $K_{2}$ are taken from Ref. [18] (Fig. 21), and the magnetic coherence length was calculated by taking $B=1 \mathrm{~T}$ and the values of $\chi_{a}$ given in Ref. [19] (Fig. 22).

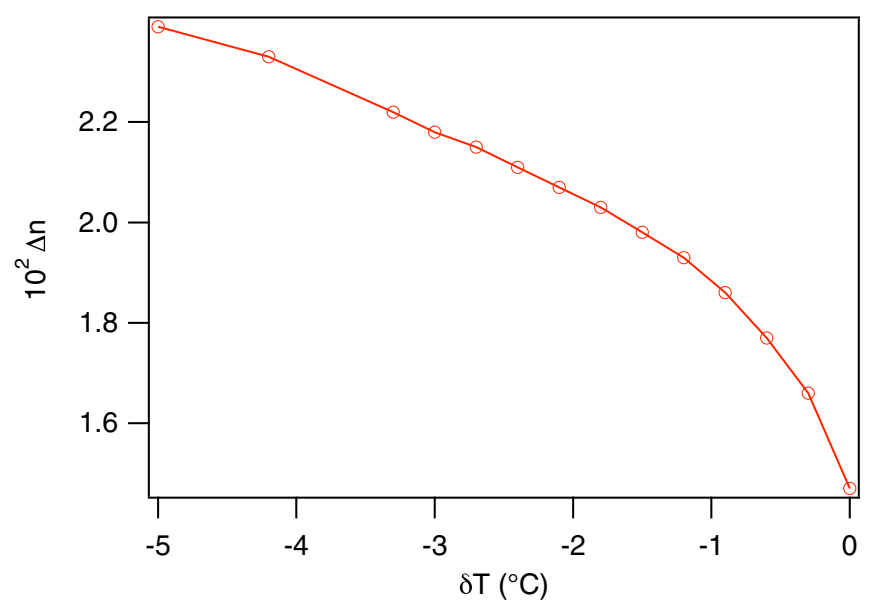

FIG. 20. Birefringence as a function of temperature at $\lambda=$ $633 \mathrm{~nm}$. 


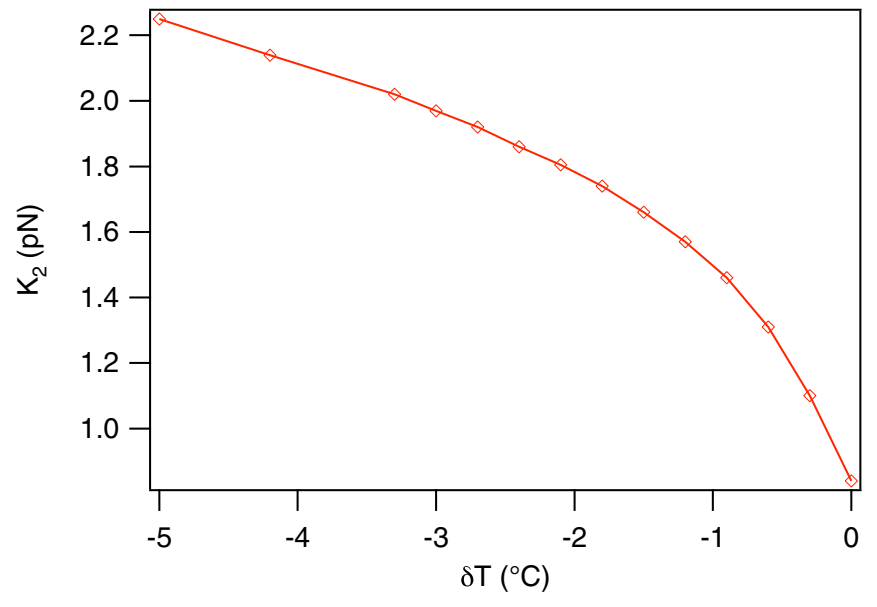

FIG. 21. Twist constant as a function of temperature.

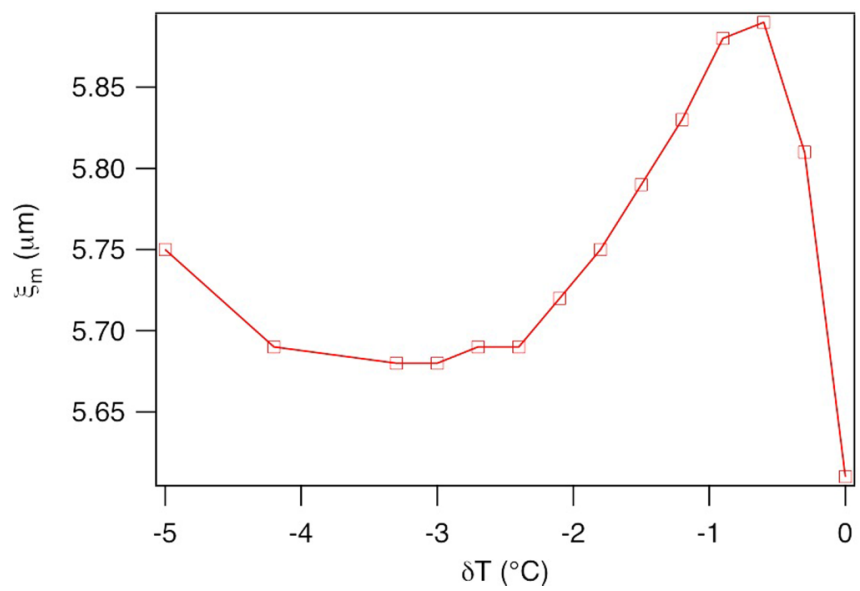

FIG. 22. Magnetic coherence length as a function of temperature.
[1] B. Jerome, Surface effects and anchoring in liquid crystals, Rep. Prog. Phys. 54, 391 (1991).

[2] P. Oswald and P. Pieranski, Nematic and Cholesteric Liquid Crystals: Concepts and Physical Properties Illustrated by Experiments (Taylor \& Francis, CRC Press, Boca Raton, FL, 2005).

[3] G. Carbone and C. Rosenblatt, Polar Anchoring Strength of a Tilted Nematic: Confirmation of the Dual Easy Axis Model, Phys. Rev. Lett. 94, 057802 (2005).

[4] S. Slussarenko, A. Murauski, T. Du, V. Chigrinov, L. Marrucci, and E. Santamato, Tunable liquid crystal q-plates with arbitrary topological charge, Opt. Express 19, 4085 (2011).

[5] A. Martinez, H. C. Mireles, and I. I. Smalyukh, Large-area optoelastic manipulation of colloidal particles in liquid crystals using photoresponsive molecular surface monolayers, Proc. Natl. Acad. Sci. USA 108, 20891 (2011).

[6] S. Hernández-Navarro, P. Tierno, J. A. Farrera, J. Ignés-Mullol, and F. Sagués, Reconfigurable swarms of nematic colloids controlled by photoactivated surface patterns, Angew. Chem. 126, 10872 (2014).

[7] V. G. Chigrinov, V. M. Kozenkov, and H.-S. Kwok, Photoalignment of Liquid Crystalline Materials: Physics and Applications, Wiley SID series in display technology (Wiley, Chichester, England/Hoboken, NJ, 2008).

[8] V.ladimir G. Chigrinov, Photoaligning and photopatterning-a new challenge in liquid crystal photonics, Crystals 3, 149 (2013).

[9] K. Ichimura, Y. Suzuki, T. Seki, A. Hosoki, and K. Aoki, Reversible change in alignment mode of nematic liquid crystals regulated photochemically by command surfaces modified with an azobenzene monolayer, Langmuir 4, 1214 (1988).

[10] G. Barbero and V. Popa-Nita, Model for the planar-homeotropic anchoring transition induced by trans-cis isomerization, Phys. Rev. E 61, 6696 (2000).

[11] G. Barbero, L. R. Evangelista, and L. Komitov, Photomanipulation of the anchoring strength of a photochromic nematic liquid crystal, Phys. Rev. E 65, 041719 (2002).
[12] H. Nadasi, R. Stannarius, A. Eremin, A. Ito, K. Ishikawa, O. Haba, K. Yonetake, H. Takezoe, and F. Araoka, Photomanipulation of the anchoring strength using a spontaneously adsorbed layer of azo dendrimers, Phys. Chem. Chem. Phys. 19, 7597 (2017).

[13] K. Ichimura, Photoalignment of liquid-crystal systems, Chem. Rev. 100, 1847 (2000).

[14] M. O'Neill and S. M. Kelly, Photoinduced surface alignment for liquid crystal displays, J. Phys. D: Appl. Phys. 33, R67 (2000).

[15] Y. Yi, M. J. Farrow, E. Korblova, D. M. Walba, and T. E. Furtak, High-sensitivity aminoazobenzene chemisorbed monolayers for photoalignment of liquid crystals, Langmuir 25, 997 (2009).

[16] P. S. Noonan, A. Shavit, B. R. Acharya, and D. K. Schwartz, Mixed alkylsilane functionalized surfaces for simultaneous wetting and homeotropic anchoring of liquid crystals, ACS Appl. Mater. Interfaces 3, 4374 (2011).

[17] B. S. Scheuble, G. Weber, and R. Eidenschink, Liquid crystalline cyclohexylcarbonitriles: Properties of single compounds and mixtures, Proc. Eurodisplay Paris 65 (1984).

[18] P. Oswald, G. Poy, and A. Dequidt, Lehmann rotation of twisted bipolar cholesteric droplets: Role of Leslie, Akopyan, and Zel'dovich thermomechanical coupling terms of nematodynamics, Liq. Cryst. 44, 969 (2017).

[19] P. Oswald, G. Poy, and F. Vittoz, Fréedericksz transition under electric and rotating magnetic field: Application to nematics with negative dielectric and magnetic anisotropies, Liq. Cryst. 44, 1223 (2017).

[20] L. Faget, S. Lamarque-Forget, P. Martinot-Lagarde, P. Auroy, and I. Dozov, Anticonical anchoring and surface transition in a nematic liquid crystal, Phys. Rev. E 74, 050701(R) (2006).

[21] G. Barbero and R. Barberi, Critical thickness of a hybrid aligned nematic liquid crystal cell, J. Phys. 44, 609 (1983).

[22] P. Oswald, Easy axis memorization with active control of the azimuthal anchoring energy in nematic liquid crystals, Europhys. Lett. 107, 26003 (2014). 